\title{
Parallel interactive retrieval of item and associative information from event memory
}

\author{
Gregory E. Cox ${ }^{\mathrm{a}, *}$, Amy H. Criss ${ }^{\mathrm{a}}$ \\ ${ }^{a}$ Department of Psychology, 430 Huntington Hall, Syracuse University, Syracuse, NY 13244-2340
}

\begin{abstract}
Memory contains information about individual events (items) and combinations of events (associations). Despite the fundamental importance of this distinction, it remains unclear exactly how these two kinds of information are stored and whether different processes are used to retrieve them. We use both model-independent qualitative properties of response dynamics and quantitative modeling of individuals to address these issues. Item and associative information are not independent and they are retrieved concurrently via interacting processes. During retrieval, matching item and associative information mutually facilitate one another to yield an amplified holistic signal. Modeling of individuals suggests that this kind of facilitation between item and associative retrieval is a ubiquitous feature of human memory.
\end{abstract}

Keywords: episodic memory; memory models; associative recognition; systems factorial technology; Bayesian statistics

\section{Introduction}

Memory contains information about both single events-"items"- and combinations of events- "associations" (Anderson \& Bower, 1973; Murdock, 1974). Being able to store and retrieve both of these kinds of information underlies the ability to discover meaningful temporal and spatial structure in the environment (e.g., causal regularities, correlations, and event schemata; Zacks \& Tversky, 2001) and dissociations between item and associative memory are important for a variety of neurological diagnoses, including memory deficits with age (Naveh-Benjamin, 2000). Despite the fundamental nature of this distinction, it remains unclear exactly how item and associative information are stored and what processes are used to retrieve them.

Many dual-process theories (Jacoby, 1991; Yonelinas, 1997) posit that item information is retrieved primarily via a "familiarity" process while associative information can only be retrieved using an independent "recollection" process. That these processes

\footnotetext{
All data, experiment code, stimuli, and model code are freely available for download from the Open Science Framework at https://osf.io/uhejm/

${ }^{*}$ Corresponding author.

Email address: gregcox7@gmail.com (Gregory E. Cox)
} 
retrieve different kinds of information implies that item and associative information are stored separately and may be represented in qualitatively different forms accessible only to particular processes. However, arguments in favor of this view have relied on measures of recognition accuracy that are not diagnostic of the types of processes involved (Dunn, 2004, 2008; Wixted, 2007; Pratte \& Rouder, 2012) and that are only reliable under the strong assumption of item and associative independence (Curran \& Hintzman, 1995, Hillstrom \& Logan, 1997, Ratcliff et al., 1995). In contrast, item and associative memory are often correlated: Item recognition is affected by the presence of an intact association, even when it is irrelevant to the task (Tulving \& Thompson, 1973: Clark \& Shiffrin 1987) and while participants are able to separately assess memory for items and associations, they are influenced by the strength of both items and associations when doing so (Buchler et al., 2008: Aue et al. 2017). While these results still allow the possibility that item and associative retrieval rely on separate processes, they imply that such processes are not mutually exclusive, with item and associative information being represented in the same memory store (Gillund \& Shiffrin, 1984).

Where there are differences between item and associative retrieval, they are most clearly found in retrieval dynamics: People can discriminate between studied and unstudied items faster than they can distinguish learned from unlearned pair associations (Gronlund \& Ratcliff, 1989, Rotello \& Heit, 2000, Nobel \& Shiffrin, 2001). While this delay has been attributed to an independent "recall-to-reject" process for associative retrieval (Rotello \& Heit 2000), this account predicts that partial cues will aid associative recognition (i.e., using a singly presented word to retrieve its studied associate), but in fact they do not (Gronlund \& Ratcliff, 1989). Paradoxically, these results are more consistent with a strong interaction between item and associative retrieval in which item information serves to "gate" associative retrieval by providing a baseline or context against which later associative information is judged (Hockley, 1991, Criss \& Shiffrin, 2005: Cox \& Shiffrin in press). A gating mechanism also explains why focusing on items impairs associative memory, but focusing on associations has no negative impact on item memory (Hockley \& Cristi, 1996a) and why associative interference occurs only among pairs comprised of the same types of items (Criss \& Shiffrin, 2004; Aue et al. 2012). Once again, these interactions imply not just that item and associative information are stored in the same memory structure, but that the processes used to retrieve them are not independent.

While there appear to be differences in the dynamics with which item and associative information are retrieved, and while it appears these two kinds of information are related in some sense, it remains unclear what the nature of these dynamic differences and relationships are. They may result from two independent retrieval processes operating on correlated memory structures, from interactions during retrieval itself, from some combination of these, or some even more exotic form of interaction. A set of experimental and analytical tools known as Systems Factorial Technology (SFT; Townsend \& Nozawa, 1995) is designed to address exactly these questions. Applying these tools to the study of long-term memory has, however, proven difficult due to various technical limitations. In this study, we overcome these limitations. Based on converging evidence from qualitative properties of retrieval dynamics as measured by SFT and from quantitative modeling of individual participants, we show that item and associative information are retrieved concurrently and that they are not independent, 
nor are independent processes used to retrieve them. After describing our experimental methods, we explain how we applied SFT analyses and individual modeling to derive these conclusions. Finally, we discuss how our results place strong constraints on future theory development and have important implications for understanding how event memory is related to long-term learning.

\section{Methods}

We measured the dynamics of item and associative retrieval in a recognition paradigm that requires retrieval of both kinds of information. After studying a list of pairs, e.g., $\mathrm{AB}, \mathrm{CD}, \mathrm{EF}$, etc., participants must later discriminate between intact studied pairs and three kinds of foil pairs. We denote intact studied pairs, like $\mathrm{AB}, I^{+} A^{+}$pairs since both the items in the pair $(I)$ and the association between the items $(A)$ match what was studied $\left({ }^{+}\right) . I^{+} A^{-}$pairs, often called "rearranged" pairs, are formed by exchanging items between two studied pairs, e.g., CF or ED; in this case, the items match the study situation, but the association does not. $I^{-} A^{+}$pairs are formed by replacing the items in an intact pair with similar unstudied items (e.g., A'B'); while the items may not exactly match what was studied, the relational information between them is preserved, leaving the association intact. $I^{-} A^{-}$pairs are formed by performing both of these operations (e.g., C'F'). Thus, neither item nor associative information is sufficient on its own to identify $I^{+} A^{+}$pairs-both item and associative information must be retrieved. This can be contrasted with the studies reviewed in the Introduction in which the presence of one or more unstudied items entails that the association is also unstudied.

To be able to apply the tools of SFT, we also separately vary the strength of each kind of information in memory, yielding both high and low associative strength, $A_{H}$ and $A_{L}$, and high and low item strength, $I_{H}, I_{L}$, for all pair types (as shown in Figure 2). As described below, these strength manipulations allow us to compute one of the critical statistics of SFT which can enable us to determine the nature of the processes by which item and associative information are retrieved and the extent to which they interact (for an overview and tutorial on SFT, see Houpt et al., 2014).

\subsection{Participants}

135 Syracuse University students took part in this experiment in exchange for course credit after providing informed consent in accord with local Institutional Review Board policy.

\subsection{Materials}

Stimuli consisted of indoor and outdoor scene images derived from two image sets (Goh et al., 2004, Konkle et al., 2010). The images were first screened to remove any legible writing (to preclude this as a strategy to remember particular images) as well as people (since these were particularly salient relative to other scene content). We then selected a subset of these images that would be most suitable for the present design, namely, images that would be easily distinguished from one another while, at the same time, being roughly symmetrical such that the left and right halves were similar. 
To that end, we defined the similarity of images/image halves using the color histogram. We converted the RGB pixel values in each image to the perceptually-based CIELAB colorspace, defined by three dimensions: lightness $L^{*}$ (ranging from 0 to 100 ), red-green chromaticity $a^{*}$ (ranging from 0 to 360 degrees), and blue-yellow chromaticity $b^{*}$ (ranging from 0 to 360 degrees). Histogram bins were defined by dividing $L^{*}$ into 8 equal-sized bins and $a^{*}$ and $b^{*}$ each into 16 equal-sized bins. Similarity between any two images $i$ and $j$ was measured using the symmetrized KL divergence between their color histograms, $F_{i}$ and $F_{j}$ :

$$
K L_{i j}=\sum_{l, a, b} F_{i}(l, a, b) \log \frac{F_{i}(l, a, b)}{F_{j}(l, a, b)}+\sum_{l, a, b} F_{j}(l, a, b) \log \frac{F_{j}(l, a, b)}{F_{i}(l, a, b)}
$$

which is 0 when $F_{i}$ and $F_{j}$ are identical and increases as the histograms become more dissimilar. We also computed the divergence between the color histograms of the left and right halves of each image $i$ in the set, $K L_{i_{L} i_{R}}$. The "goodness" $g_{i}$ of an image $i$ was defined as the ratio between the minimum inter-image divergence and the intra-image divergence, $g_{i}=\min K L_{i j} / K L_{i_{L} i_{R}}$, which is large when the image is highly dissimilar to all other images and/or highly symmetrical and is small when the image is very similar to other images and/or very asymmetrical. We then selected the images with the top 512 "goodness" values for use in the experiment. Images were down-sampled to a uniform size of $256 \times 256$ pixels.

\subsection{Design}

Each study/test list was comprised of 16 unique pairs of image halves, divided evenly into four strength conditions defined by the factorial combination of high and low item strength and high/low associative strength, as summarized in Table 1 . High associative strength pairs were presented 3 times during the study list while low associative strength pairs were presented only once during study. The image halves in a low item strength pair appeared only as part of the study pair. For high item strength pairs, each image half from the pair was presented twice paired with itself (e.g., AA and BB). In total, the study list comprised 64 trials: 8 low strength pair presentations, 24 high strength pair presentations ( 8 pairs repeated 3 times), and 32 self-pairings of an image half ( 2 presentations each of the 2 halves of 8 high item strength pairs). Test lists consisted of 16 pairs, summarized in Table 2, with examples of each pair type shown in Figure 1. Thus, each study/test block provides one observation of each cell in the double factorial design.

\subsection{Procedure}

The experiment was implemented in PsychoPy (Peirce, 2007). Participants engaged in 10 to 11 study/test blocks depending on how many they could complete in an hour. The images used to construct the study and test lists were randomly sampled without replacement from the stimulus pool for each study/test block.

Prior to each block, participants were told that they would be shown sets of image pairs and that they should try to remember which images appeared together. Study pairs were then presented on a white background for 2 seconds at a time, with a $0.5 \mathrm{sec}-$

ond blank between each pair presentation. Presentation order was randomized under 
Table 1: Design of pairs used in each study list, from which test lists were constructed (see Table 2). Numbers refer to a particular image (of which only the left or right half was studied) within a study-test block; different images were randomly drawn from the stimulus pool without replacement for each study-test block, such that the images labeled "1", "2", etc., differed for each block. High $(\mathrm{H})$ associative strength pairs were shown three times during study, while low (L) associative strength pairs were shown only once. High (H) item strength pairs had each of their component items shown paired with themselves (e.g., [1, 1] and [2, 2]) twice during the study phase.

\begin{tabular}{cll}
\hline Images in pair & Item strength & Associative strength \\
\hline 1,2 & $\mathrm{H}$ & $\mathrm{H}$ \\
3,4 & $\mathrm{H}$ & $\mathrm{L}$ \\
5,6 & $\mathrm{H}$ & $\mathrm{H}$ \\
7,8 & $\mathrm{H}$ & $\mathrm{H}$ \\
9,10 & $\mathrm{H}$ & $\mathrm{L}$ \\
11,12 & $\mathrm{H}$ & $\mathrm{L}$ \\
13,14 & $\mathrm{~L}$ & $\mathrm{H}$ \\
15,16 & $\mathrm{~L}$ & $\mathrm{~L}$ \\
17,18 & $\mathrm{~L}$ & $\mathrm{H}$ \\
19,20 & $\mathrm{~L}$ & $\mathrm{H}$ \\
21,22 & $\mathrm{~L}$ & $\mathrm{~L}$ \\
23,24 & $\mathrm{~L}$ & $\mathrm{~L}$ \\
25,26 & $\mathrm{H}$ & $\mathrm{H}$ \\
27,28 & $\mathrm{H}$ & $\mathrm{L}$ \\
29,30 & $\mathrm{~L}$ & $\mathrm{H}$ \\
31,32 & $\mathrm{~L}$ & $\mathrm{~L}$ \\
\hline
\end{tabular}

Table 2: Design of each test list. Numerals refer to the same images in the study design in Table 1 with apostrophes denoting the unstudied half of the image labeled by the numeral. Participants were instructed to give a positive response to pairs in the upper left quadrant $\left(I^{+} A^{+}\right.$pairs, shown here with a gray background) and to give a negative response to other types of test pair. Because different images were randomly sampled (without replacement) from the stimulus pool for each study-test block, the images labeled "1", "2", etc. differed for each block. Thus, this table represents the experimenter's knowledge of the design and could not be inferred by the participant.

Associative strength

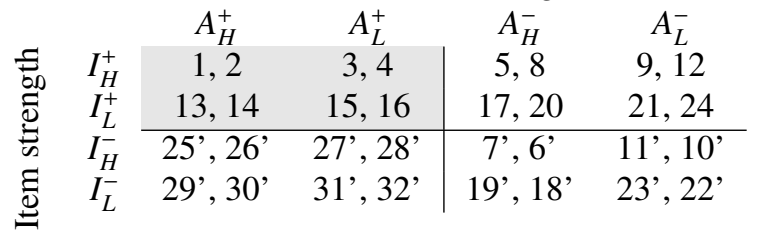




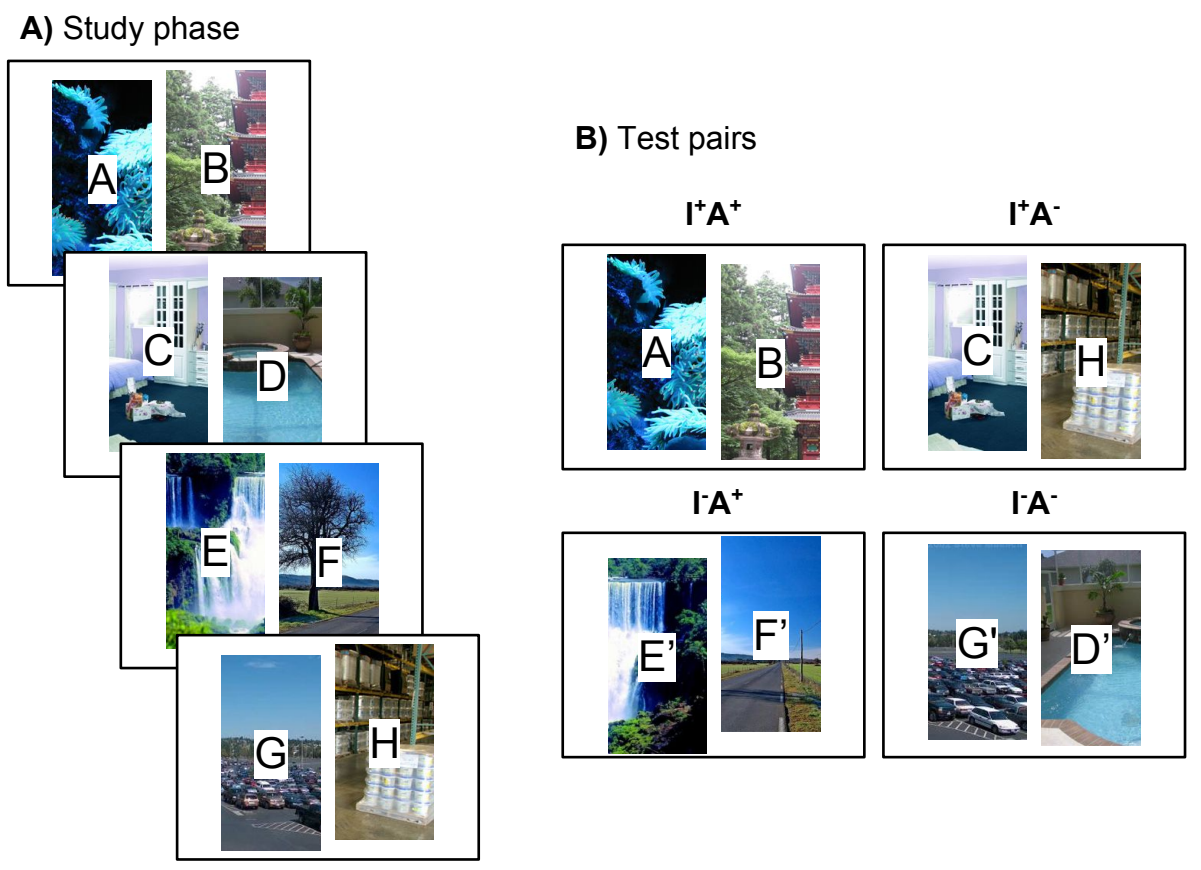

Figure 1: Examples of four image pairs shown at study (A) and how they can be used to construct the four types of test pair (B). In the experiment, no letters were superimposed on the images-this is merely for illustration. Apostrophes denote the unstudied half of an image, e.g., E' is the unstudied half of image $\mathrm{E}$. 
the constraint that two successive pairs did not contain any of the same image halves. The two image halves subtended approximately 3 degrees of visual angle, with approximately 0.5 degrees of blank space between them. Although the image pairs were centered horizontally on the screen, the left and right halves were independently offset from vertical center by random values sampled uniformly from [-0.25, 0.25] degrees of visual angle. This offset, illustrated in Figure 1 , served two purposes: first, to emphasize that the two image halves were not meant to be treated as part of the same image; second, to avoid visual masking between successive pair presentations. Assignment of left/right position within a pair was the same each time a pair was presented in that study/test block.

After presentation of all study pairs, participants were informed that they would be shown another set of image pairs and should give a positive response only to pairs of images that had appeared together at the same time on the most recent list (i.e., $I^{+} A^{+}$ pairs). Positive and negative responses were randomly mapped to the "F" or "J" keys for each participant. Participants were instructed to respond as quickly and accurately as possible. Test instructions appeared on screen for a minimum of 15 seconds, after which participants could press "enter" to proceed to the test list. Each test trial began with a fixation cross centered on the screen for 0.5 seconds followed by presentation of the test pair (which followed the same sizing and random vertical offset procedure used during study). The test pair remained on screen until a response was made, after which feedback was given. Participants were told whether their response was "correct" or "incorrect", with font color green if correct and red if incorrect. Regardless of correctness, if the response was made in under $300 \mathrm{~ms}$, feedback included a statement to "please take more time to respond" and if the response was made in over 4 seconds, feedback included a statement to "please try to respond more quickly". Feedback appeared for a minimum of 1 second, an additional 0.5 seconds if the participant was incorrect, and an additional 3 seconds if the response was too fast. A random time sampled at uniform between 0.25 and 0.75 seconds preceded the onset of the next test trial to prevent participants from entraining to a constant presentation rate and producing rhythmic response time artifacts.

\section{Results}

Prior to analysis, we excluded trials in which the response time was shorter than $200 \mathrm{~ms}$ ( 47 out of 21,369 trials) or longer than $5 \mathrm{~s}$ (127 trials). Excessively long response times are likely to reflect processes beyond those involved in recognizing the test pair, particularly distractions, while excessively short response times could not have resulted from processing the test pair at all. Based on the remaining trials, we excluded participants who failed to give a higher rate of positive responses to intact pairs than to foils or who did not give any correct responses in at least one condition in order to ensure that our conclusions were based on individuals who understood the task instructions. All subsequent analyses were carried out on the remaining 18,760 trials from 118 participants.

Mean proportion of positive ("yes") responses is given in Table 3 and median correct RT in Table 4 for each combination of item and associative strength. A 4 (item 
Table 3: Observed mean proportion of "yes" responses (with standard deviations in parentheses) for each combination of item and associative strength. Shaded cells indicate $I^{+} A^{+}$pairs, which should be given a positive response.

\begin{tabular}{|c|c|c|c|c|}
\hline & \multicolumn{4}{|c|}{ Associative strength } \\
\hline & $A_{H}^{+}$ & $A_{L}^{+}$ & $A_{H}^{-}$ & $A_{L}^{-}$ \\
\hline$I_{H}^{+}$ & $0.75(0.19)$ & $0.54(0.19)$ & $0.37(0.20)$ & $0.34(0.19)$ \\
\hline$I_{L}^{+}$ & $0.72(0.23)$ & $0.46(0.19)$ & $0.31(0.17)$ & $0.22(0.16)$ \\
\hline$I_{H}^{-}$ & $0.26(0.17)$ & $0.21(0.15)$ & $0.15(0.15)$ & $0.16(0.15)$ \\
\hline$I_{L}^{-}$ & $0.27(0.17)$ & $0.22(0.13)$ & $0.14(0.15)$ & $0.12(0.12)$ \\
\hline
\end{tabular}

Table 4: Observed median correct response time (in seconds, with standard deviations in parentheses) for each combination of item and associative strength. Shaded cells indicate $I^{+} A^{+}$pairs, which should be given a positive response.

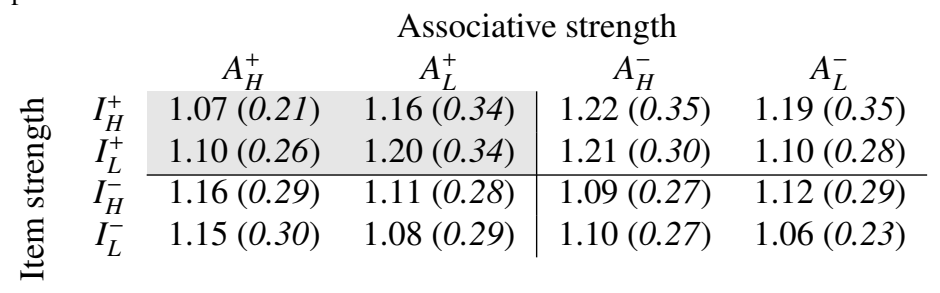

strength) $\times 4$ (associative strength) within-subjects ANOVA on the proportion of positive responses finds main effects of item strength $(F(3,351)=298.4, p \sim 0)$ and associative strength $(F(3,351)=238.3, p \sim 0)$ as well as a significant interaction $(F(9,1053)=42.4, p \sim 0)$. Using the same $4 \times 4$ ANOVA to analyze median correct RT, we again find main effects of item strength $(F(3,351)=10.44, p \sim 0)$ and associative strength $(F(3,351)=3.01, p \approx 0.03)$ as well as a significant interaction $(F(9,1053)=8.99, p \sim 0)$.

The above analyses merely establish the effectiveness of our experimental manipulations. Inspection of Tables 3 and 4 suggests several important questions that, if answered, would provide a strong characterization of the processes underlying item and associative memory:

1. As the strength of either item or associative information is increased,$I^{+} A^{+}$pairs are correctly accepted more often and more quickly. As the strength of either item or associative information is decreased, foil pairs are correctly rejected more often and more quickly, albeit this difference is less pronounced for $\mathrm{I}^{-} A^{+}$ and $I^{-} A^{-}$pairs than for $I^{+} A^{-}$pairs.

(a) Does this indicate any interactions between item and associative memory processes?

(b) If so, do those interactions occur during retrieval, are they a byproduct of how information is stored in memory, or both?

2. $I^{+} A^{+}$pairs are accepted more often and faster than either $I^{-} A^{+}$or $I^{+} A^{-}$pairs. Similarly, $I^{-} A^{-}$pairs are correctly rejected more often and more quickly than $I^{+} A^{-}$or $I^{-} A^{+}$pairs. How much does it help to have two sources of matching information (both items and association) rather than just one? How much does 


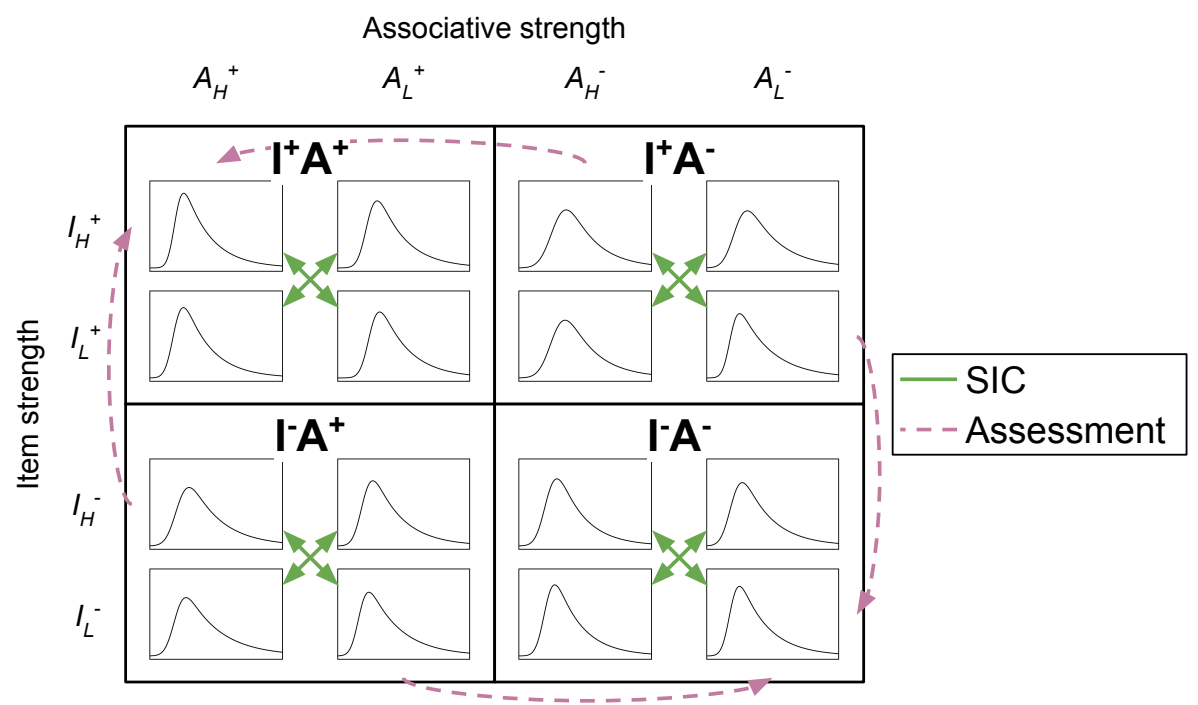

Figure 2: Double factorial manipulation of item and associative strength, allowing estimation of response time distributions in each cell (with probability density on the vertical axis and time on the horizontal axis). Colored lines indicate the distributions used to compute qualitative signatures of retrieval processes. The survivor interaction contrast (SIC) function examines interactions in retrieval speed by comparing the four distributions within each pair type, while the assessment function examines how matching and mismatching item and associative information is combined across pair types.

it help to have two sources of mismatching information? What do these interactions imply about how matching and mismatching information is treated during retrieval?

Each of these questions is answered in the analyses below, in which we use the tools of SFT and individual modeling to characterize the processes underlying item and associative memory.

\section{Analysis}

Our analysis begins by first obtaining robust estimates of the response time distributions in each condition. We use the estimated RT distributions to compute the statistics needed for SFT: the survivor interaction contrast (SIC) functions and the capacity assessment functions, described in detail below and summarized schematically in Figure 2. The SIC functions answer question 1a pertaining to the presence of interactions between item and associative memory. The assessment functions answer question 2 regarding how matching and mismatching item and associative information are combined. Finally, we use modeling of individuals to both validate the conclusions drawn from SFT and to answer question $1 \mathrm{~b}$, regarding the loci of interactions between item and associative memory. 


\subsection{Estimating RT distributions}

In contrast to most applications of SFT (and response time studies generally), we have a large number of participants but relatively few observations per participant per condition. We therefore take a parametric Bayesian approach to estimating RT distributions, which has the additional benefit of allowing for robust statistical tests of the resulting SFT measures (for background on Bayesian data analysis, see Kruschke, 2015).

\subsubsection{Parameters}

Each subject $s$ is associated with a total of $4 \times 4 \times 2=32$ RT distributions defined by the factorial combination of item strength $i$, associative strength $j$, and positive ("yes")/negative ("no") response $r$. We assume that all of these distributions are Ex-Gaussian with parameters $\hat{m}_{i j r s}$ (mean of the Gaussian component), $\hat{s}_{i j r s}$ (standard deviation of the Gaussian component), and $\hat{t}_{i j r s}$ (shape of the exponential component) and cumulative distribution function

$$
F_{i j r s}(x)=\Phi\left(\frac{x-\hat{m}_{i j r s}}{\hat{s}_{i j r s}}\right)-\Phi\left(\frac{x-\hat{m}_{i j r s}}{\hat{s}_{i j r s}}-\frac{\hat{s}_{i j r s}}{\hat{t}_{i j r s}}\right) \exp \left(\frac{\hat{s}_{i j r s}^{2}}{2 \hat{t}_{i j r s}^{2}}-\frac{x-\hat{m}_{i j r s}}{\hat{t}_{i j r s}}\right)
$$

where $\Phi(\cdot)$ is the standard normal cumulative distribution function. The Ex-Gaussian has been found to provide a good description of RT distributions in recognition memory (Ratcliff \& Murdock, 1976) and can accommodate a variety of distribution shapes. In addition to these parameters, we estimate the probability $\hat{\theta}_{i j s}$ that participant $s$ makes a positive response to a pair with item strength $i$ and associative strength $j$.

\subsubsection{Bayesian model}

All of the above parameters are estimated simultaneously according to the hierarchical Bayesian model described in Appendix C (Rouder et al. 2005). The model was implemented in JAGS (Plummer, 2013), which was used to obtain 10,000 posterior samples split over 10 parallel chains after 2000 iterations of "burn-in" each. By obtaining a posterior distribution over RT distributions, we obtain posterior distributions over quantities derived from these distributions, namely, the key statistics needed in Systems Factorial Technology.

\subsubsection{Posterior RT distributions}

For each of the 10,000 posterior samples, we obtained an estimate of the RT distributions across all pair types by using the Ex-Gaussian RT distributions derived from the mear 1 subject parameters in that sample. For each level of item strength $i$, associative strength $j$, and response $r$, the mean subject parameters for sample $n$ are given

\footnotetext{
${ }^{1}$ Other measures of central tendency, like the median and mode, give qualitatively identical results to those reported here.
} 
by:

$$
\begin{aligned}
\bar{\theta}_{i j}[n] & =\operatorname{Mean}_{s}\left[\hat{\theta}_{i j s}[n]\right] \\
\bar{m}_{i j r}[n] & =\operatorname{Mean}_{s}\left[\hat{m}_{i j r s}[n]\right] \\
\bar{s}_{i j r}[n] & =\operatorname{Mean}_{s}\left[\hat{s}_{i j r s}[n]\right] \\
\bar{t}_{i j r}[n] & =\operatorname{Mean}_{s}\left[\hat{t}_{i j r s}[n]\right]
\end{aligned}
$$

The estimate for the RT distribution given item strength $i$, associative strength $j$, and response $r$ is then found by plugging the sampled modal parameters into Equation 1 . yielding the distribution function

$$
F_{i j r}[n](x)=\Phi\left(\frac{x-\bar{m}_{i j r}[n]}{\bar{s}_{i j r}[n]}\right)-\Phi\left(\frac{x-\bar{m}_{i j r}[n]}{\bar{s}_{i j r}[n]}-\frac{\bar{s}_{i j r}[n]}{\bar{t}_{i j r}[n]}\right) \exp \left(\frac{\bar{s}_{i j r}[n]^{2}}{2 \bar{t}_{i j r}[n]^{2}}-\frac{x-\bar{m}_{i j r}[n]}{\bar{t}_{i j r}[n]}\right)
$$

as well as the probability $P_{i j}[n]=\bar{\theta}_{i j}[n]$, the modal probability of giving a positive response. The result is 10,000 posterior samples of the group-level RT distributions and response probabilities for each combination of item strength, associative strength, and response type. These samples, in turn, yield samples of the SFT statistics needed to identify the type of retrieval architecture operating in this task, as we now describe.

\subsection{Systems factorial analysis}

While previous studies have found that item information is retrieved more quickly than associative information, as discussed in the Introduction, it remains unclear how these two retrieval processes are related. The tools of Systems Factorial Technology (SFT) are ideally suited to address this issue. SFT makes no a priori commitments to any of the many possible arrangements of retrieval processes and interactions that could be used in this paradigm, instead allowing for a simultaneous comparison against all possibilities. It may be that item and associative information are retrieved simultaneously (though not necessarily at the same rate), called parallel processing, or it may be that one type of information (say, associative) is not retrieved until the first retrieval process is finished, called serial processing. If either item or associative information can be used to make a response, processing is called "self-terminating". If, however, both types of information must be retrieved to generate a response, processing is "exhaustive". Two responses are made in this paradigm - either a positive "yes" response or a negative "no" response-so if one type of response requires exhaustive processing, then the other logically entails self-terminating processing (otherwise there would be a non-trivial probability of never making a response).

We conceive of interactions between item and associative retrieval as a kind of pooling in which match and/or mismatch information is mutually reinforced, as shown in Figure 3 A. If positive evidence from both item and associative retrieval is pooled into a holistic "match", we say that positive responses are facilitated whereas negative responses are inhibited, and vice versa if negative evidence is pooled into a holistic "mismatch". In the extreme case, both match and mismatch information might be pooled from both sources, making them effectively inseparable, termed "coactive" processing. 


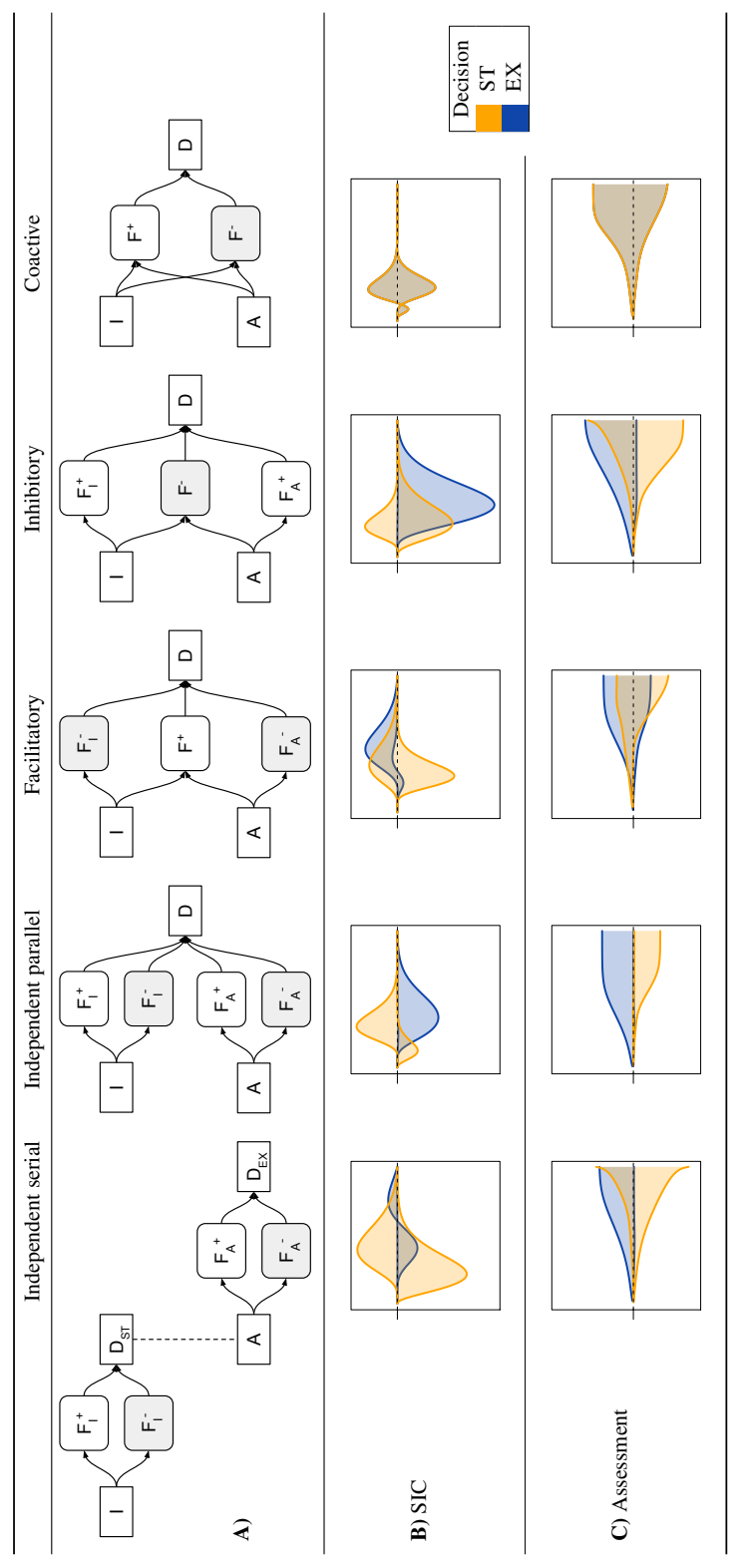

Figure 3: Different retrieval architectures yield different qualitative predictions for response dynamics. A) Schematic depictions of possible retrieval architectures. Match $\left(F^{+}\right)$and mismatch $\left(F^{-}\right)$information from item (I) and associative (A) retrieval processes are fed into a decision rule (D) which may either be selfterminating (ST) or exhaustive (EX). Depending on the decision rule and retrieval architecture, the survivor interaction contrast (SIC; B) and assessment functions $(\mathbf{C})$ can take different qualitative forms. For illustrative purposes, $\mathbf{B}$ and $\mathbf{C}$ depict an "envelope" of possible forms for each architecture ranging from the greatest possible positive deflection to the greatest possible negative deflection, with time along the horizontal axes. Labels on the top row are with respect to positive responses, such that facilitatory processing for positive responses is inhibitory processing for negative responses. 
Note that any kind of interaction of this form entails parallel processing since even if one process begins earlier than the other, they must eventually operate concurrently in order to pool their information. This kind of parallel processing falls under the heading of "continuous-flow" systems (Schweickert, 1989; Miller, 1993; , Townsend \& Fikes, 1995, Liu, 1996), one example of which is the well-known "cascade" model (McClelland. 1979, Ashby, 1982) in which the output from early stages of processing serves as input to subsequent stages.

The key insight in SFT is that all of these different kinds of processing will leave qualitatively different signatures in the response time (RT) distributions participants produce. By comparing the qualitative forms of these signatures to those allowed by each retrieval architecture, we characterize how item and associative information are retrieved and how they interact. In situations where the experimental manipulation selectively influences only one process, analytic methods alone can be used to derive predictions for these signatures (Townsend \& Nozawa 1995). We could not guarantee selective influence in our applications, however. Consider, for example, that repeating a pair to enhance the strength of the stored association also entails repeating the two items in the pair, likely increasing their strength as well, or that repeating items in isolation (AA or $\mathrm{BB}$ ) may interfere with their association (AB). In addition, error rates tend to be higher in our study than in those to which SFT is typically applied. We therefore obtained predictions for the SFT measures using a combination of simulations (Diederich \& Busemeyer, 2003; Fific et al., 2008, Eidels et al., 2011) and analytic methods (Townsend \& Thomas, 1994), as described in Appendix A. The resulting predictions allow for both a large proportion of errors (from near 0\% to 96\%) and violations of selective influence; while we lose the statistical power available with low error rates and selective influence, we can be confident that we have provided a fair qualitative characterization of each possible retrieval architecture.

\subsubsection{Interactions between item and associative memory}

As shown in Figure 2, the Survivor Interaction Contrast (SIC) function compares RT distributions within each pair type in order to characterize how varying the strengths of item and associative information affect retrieval speed for each type of pair. Specifically, the SIC measures whether the effect of changing the strength of one type of information depends on how strong the other information source is (e.g., is going from $I_{L}^{+} A_{L}^{+}$to $I_{H}^{+} A_{L}^{+}$different than going from $I_{L}^{+} A_{H}^{+}$to $I_{H}^{+} A_{H}^{+}$?). In this sense, it is analogous to a mean interaction contrast in Analysis of Variance, only instead of just the mean response times, the contrast is applied to the entire distribution.

For example, increasing the strength of either item or associative information should lead to faster correct responses to $I^{+} A^{+}$pairs, regardless of what kind of retrieval process is involved. This speedup is reflected in the survivor function ${ }^{2}$ of the response time distribution: For all times $t, S_{I_{H}^{+} A_{H}^{+}}(t) \leq S_{I_{H}^{+} A_{L}^{+}}(t), S_{I_{H}^{+} A_{H}^{+}}(t) \leq S_{I_{L}^{+} A_{H}^{+}}(t), S_{I_{H}^{+} A_{H}^{+}}(t) \leq$ $S_{I_{L}^{+} A_{L}^{+}}(t), S_{I_{H}^{+} A_{L}^{+}}(t) \leq S_{I_{L}^{+} A_{L}^{+}}(t)$, and $S_{I_{L}^{+} A_{H}^{+}}(t) \leq S_{I_{L}^{+} A_{L}^{+}}(t)$, where each $S_{i j}(t)=1-F_{i j}(t)$ is the survivor function of the correct response times to pairs with item strength $i$ and associative strength $j$. The SIC for $I^{+} A^{+}$pairs then asks whether going from $I_{L}^{+} A_{L}^{+}$to

\footnotetext{
${ }^{2}$ The survivor function is one minus the cumulative distribution function.
} 
$I_{H}^{+} A_{L}^{+}$-increasing item strength when associative strength is low-produces a different speedup than going from $I_{L}^{+} A_{H}^{+}$to $I_{H}^{+} A_{H}^{+}$-increasing item strength when associative strength is high. Similarly, for pairs that participants should reject as foils $\left(I^{+} A^{-}, I^{-} A^{+}\right.$, and $\left.I^{-} A^{-}\right)$, reducing the strength of either item or associative information should lead to faster correct responses $\left(S_{I_{L}^{-} A_{L}^{-}}(t) \leq S_{I_{H}^{-} A_{L}^{-}}(t)\right.$, etc.) and the SIC for those pairs asks whether this speedup depends on the strength of the other source of information. Formally, the SIC function for each type of pair is given by

$$
\begin{aligned}
S I C_{I^{+} A^{+}}(t) & =\left[S_{I_{L}^{+} A_{L}^{+}}(t)-S_{I_{L}^{+} A_{H}^{+}}(t)\right]-\left[S_{I_{H}^{+} A_{L}^{+}}(t)-S_{I_{H}^{+} A_{H}^{+}}(t)\right] \\
S I C_{I^{+} A^{-}} & (t)=\left[S_{I_{H}^{+} A_{H}^{-}}(t)-S_{I_{H}^{+} A_{L}^{-}}(t)\right]-\left[S_{I_{L}^{+} A_{H}^{-}}(t)-S_{I_{L}^{+} A_{L}^{-}}(t)\right] \\
S I C_{I^{-} A^{+}} & (t)=\left[S_{I_{H}^{-} A_{H}^{+}}(t)-S_{I_{H}^{-} A_{L}^{+}}(t)\right]-\left[S_{I_{L}^{-} A_{H}^{+}}(t)-S_{I_{L}^{-} A_{L}^{+}}(t)\right] \\
S I C_{I^{-} A^{-}} & (t)=\left[S_{I_{H}^{-} A_{H}^{-}}(t)-S_{I_{H}^{-} A_{L}^{-}}(t)\right]-\left[S_{I_{L}^{-} A_{H}^{-}}(t)-S_{I_{L}^{-} A_{L}^{-}}(t)\right] .
\end{aligned}
$$

Depending on whether retrieval is serial or parallel, whether the decision rule is self-terminating or exhaustive, and whether or not the two retrieval processes interact, the SIC can take a variety of possible forms, as depicted in Figure 3 B. Although Appendix A contains the complete derivation these forms, to provide some intuition, we consider why the SIC can take on different forms depending on the kind of interaction between item and associative retrieval.

Independent: If item and associative retrieval are independent, they could be retrieved either in serial or in parallel. Imagine that item and associative information are retrieved in parallel and that "yes" responses are made using an exhaustive decision rule such that both processes must yield a match before a pair is recognized. As either item or associative strength is increased, recognition is faster, but because of the exhaustive decision rule, having low strength on either dimension still results in relatively slow processing; only when both types of information are high strength does a substantial speedup result. This is reflected in the $S I C_{I^{+} A^{+}}$ function for correct acceptance responses that dips below zero. On the other hand, the SIC functions for correct rejections will have positive deflections because they are self-terminating - only one source needs to mismatch in order to get a big speedup. These examples make clear that the SIC can deviate from zero even in the absence of any interactions between the two retrieval processes due to statistical facilitation (or inhibition) resulting from the stopping rule.

Facilitatory: If there are interactions between item and associative retrieval, they cannot be retrieved strictly in parallel since, as mentioned above, they must operate concurrently for at least some time in order to interact. Imagine that matching item and associative information facilitated one another, but the decision rule was still to respond "yes" only if both item and associative retrieval processes yielded a positive response. Although exhaustive processing still entails waiting for both kinds of information to be retrieved, which would ordinarily yield a negative SIC, facilitation overcomes this and allows for the SIC to be greater than zero, reflecting a true-rather than merely statistical—facilitatory interaction between item and associative retrieval. 
Inhibitory: Continuing with the example above, if match information is facilitated, this has the effect of inhibiting mismatch information in the sense that more mismatch is needed to overcome any mutually facilitatory match information. "No" responses then result from inhibitory self-terminating processing. Even though, in this example, only one process needs to yield a negative result for a "no" response to be made, the facilitation of the competitor response means that rejections are fast only when both item and associative strength are low, resulting in an SIC that is equal to or less than zero.

Coactive: If both match and mismatch information are pooled together, coactive processing results, for which it no longer makes sense to consider self-terminating or exhaustive decision rules, since there is effectively only one source of (pooled) information. It seems natural to think that coactive processing is the ultimate form of facilitatory processing and, as such, should yield strictly positive SIC's, but this is not the whole story: recall that facilitation of one response is inhibition for the other. By pooling all information, the match evidence is contaminated by mismatch evidence and vice versa. Coactive processing can thus predict a negative SIC if the evidence for a correct response is sufficiently weak. Further, even if evidence is strong and there is a positive deflection in the SIC, any such deflection will always be preceded by a, typically smaller, negative deflection, owing to the pooling of information leading to both correct and incorrect responses.

SIC's were computed for each sample of RT distributions from the Bayesian posterior (see above). Specifically, for each sample $n$, we plug in the survivor functions $S . .[n](t)=1-F . .[n](t)$ computed from Equation (2) to obtain $n=1 . .10,000$ samples of each SIC

$$
\begin{aligned}
S I C_{I^{+} A^{+}}[n](t) & =\left[S_{I_{L}^{+} A_{L}^{+}}[n](t)-S_{I_{L}^{+} A_{H}^{+}}[n](t)\right]-\left[S_{I_{H}^{+} A_{L}^{+}}[n](t)-S_{I_{H}^{+} A_{H}^{+}}[n](t)\right] \\
S I C_{I^{+} A^{-}}[n](t) & =\left[S_{I_{H}^{+} A_{H}^{-}}[n](t)-S_{I_{H}^{+} A_{L}^{-}}[n](t)\right]-\left[S_{I_{L}^{+} A_{H}^{-}}[n](t)-S_{I_{L}^{+} A_{L}^{-}}[n](t)\right] \\
S I C_{I^{-} A^{+}}[n](t) & =\left[S_{I_{H}^{-} A_{H}^{+}}[n](t)-S_{I_{H}^{-} A_{L}^{+}}[n](t)\right]-\left[S_{I_{L}^{-} A_{H}^{+}}[n](t)-S_{I_{L}^{-} A_{L}^{+}}[n](t)\right] \\
S I C_{I^{-} A^{-}}[n](t) & =\left[S_{I_{H}^{-} A_{H}^{-}}[n](t)-S_{I_{H}^{-} A_{L}^{-}}[n](t)\right]-\left[S_{I_{L}^{-} A_{H}^{-}}[n](t)-S_{I_{L}^{-} A_{L}^{-}}[n](t)\right] .
\end{aligned}
$$

We can then examine the resulting distributions over each SIC function to determine whether it has credible deflections above and/or below zero, as predicted by each possible retrieval architecture.

The observed SIC function for correct acceptance of $I^{+} A^{+}$pairs $\left(S I C_{I^{+} A^{+}}(t)\right.$, Figure 4. upper left) demonstrates a credible positive peak (95\% credible interval [CI] of the maximum is $[0.023,0.114])$ but no evidence for any negative deflections $(95 \%$ $\mathrm{CI}$ of the minimum is $[-0.015,0]$, thus including zero), ruling out any retrieval architecture that disallows positive SIC deflections (like independent parallel exhaustive or inhibitory exhaustive processing) as well as those that only allow positive deflections when accompanied by a negative deflection (like independent serial exhaustive or coactive processing), as summarized in Table 5 . The SIC's for each type of correct rejection (Figure 4) differ quantitatively but are qualitatively similar. None demonstrate any credible positive deflections $\left(95 \% \mathrm{CI}\right.$ of the maximum for $S I C_{I^{+} A^{-}}$is $[0,0.022]$, for 
$\mathrm{I}^{+} \mathrm{A}^{+}$
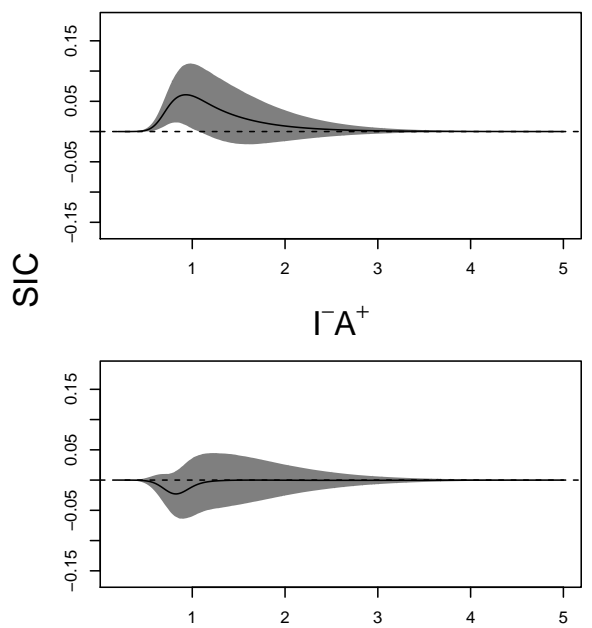

$I^{+} A^{-}$

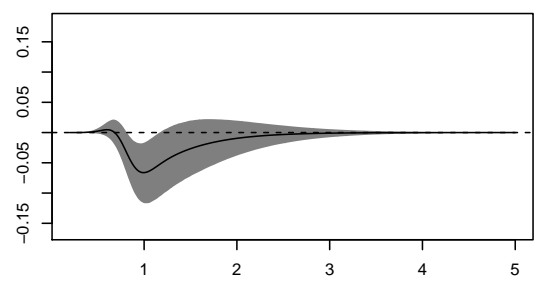

$I^{-} A^{-}$

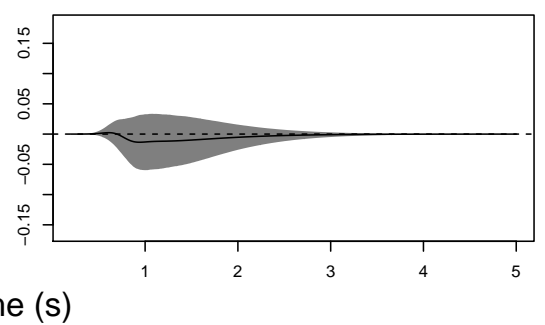

Figure 4: SIC functions for correct responses. Solid lines are posterior means while shaded regions are 95\% credible intervals.

$S I C_{I^{-} A^{+}}$is $[0,0.040]$, and for $S I C_{I^{-} A^{-}}$is [0, 0.032], all including zero), and $S I C_{I^{+} A^{-}}(t)$ demonstrates a credible negative deflection $(95 \% \mathrm{CI}$ of the minimum is [-0.117, $0.019]$ ). We can therefore rule out any retrieval arrangements that prohibit negative SIC's for negative responses (see Table 5). In answer to question 1a, the SIC's for each pair type imply that item and associative information are retrieved in parallel, not one after the other (serially), and that if there are interactions, they serve to mutually reinforce matching item and associative information (see Table 5].

\subsubsection{Combining matching and mismatching information}

While the SIC characterizes how item and associative retrieval interact as strength is varied within a pair type, a different function describes how retrieval dynamics differ between pair types. This function is the capacity assessment function, adapted from Equation 2 in Townsend \& Altieri (2012), also termed the "integration" function ( $\mathrm{Al}-$ tieri et al. 2014). The assessment function compares RT distributions when both item and associative information are congruent $\left(I^{+} A^{+}\right.$or $\left.I^{-} A^{-}\right)$to when they conflict $\left(I^{+} A^{-}\right.$ or $I^{-} A^{+} \sqrt{3}$ We can therefore compute an assessment function for both correct accep-

\footnotetext{
${ }^{3}$ In this sense, it is closely related to the "resilience function" (Little et al. 2015).
} 
tance responses $\left(A_{C A}(t)\right)$ and correct rejections $\left(A_{C R}(t)\right)$ :

$$
\begin{aligned}
& \log \left[F_{I_{H}^{+} A_{H}^{-}}^{+}(t) P_{I_{H}^{+} A_{H}^{-}}\left(1-P_{I_{H}^{-} A_{H}^{+}}\right)+F_{I_{H}^{-} A_{H}^{+}}^{+}(t) P_{I_{H}^{-} A_{H}^{+}}\left(1-P_{I_{H}^{+} A_{H}^{-}}\right)+F_{I_{H}^{+} A_{H}^{+}}^{+}(t) P_{I_{H}^{+} A_{H}^{-}} S_{I_{H}^{-} A_{H}^{+}}^{+}(t) P_{I_{H}^{-} A_{H}^{+}}\right. \\
& A_{C A}(t)=\frac{\left.+S_{I_{H}^{+} A_{H}^{-}}^{+}(t) P_{I_{H}^{+} A_{H}^{-}} F_{I_{H}^{+} A_{H}^{+}}^{+}(t) P_{I_{H}^{-} A_{H}^{+}}+F_{I_{H}^{+} A_{H}^{-}}^{+}(t) P_{I_{H}^{+} A_{H}^{-}} F_{I_{H}^{+} A_{H}^{+}}^{+}(t) P_{I_{H}^{-} A_{H}^{+}}\right]}{\log \left[F_{I_{H}^{+} A_{H}^{+}}^{+}(t) P_{I_{H}^{+} A_{H}^{+}}\right]} \\
& \log \left[F_{I_{L}^{+} A_{L}^{-}}^{-}(t)\left(1-P_{I_{L}^{+} A_{L}^{-}}\right) P_{I_{L}^{-} A_{L}^{+}}+F_{I_{L}^{-} A_{L}^{+}}^{-}(t)\left(1-P_{I_{L}^{-} A_{L}^{+}}\right) P_{I_{L}^{+} A_{L}^{-}}+F_{I_{L}^{+} A_{L}^{-}}^{-}(t)\left(1-P_{I_{L}^{+} A_{L}^{-}}\right) S_{I_{L}^{-} A_{L}^{+}}^{-}(t)\left(1-P_{I_{L}^{-} A_{L}^{+}}\right)\right. \\
& A_{C R}(t)=\frac{\left.+S_{I_{L}^{+} A_{L}^{-}}^{-}(t)\left(1-P_{I_{L}^{+} A_{L}^{-}}\right) F_{I_{L}^{-} A_{L}^{+}}^{-}(t)\left(1-P_{I_{L}^{-} A_{L}^{+}}\right)+F_{I_{L}^{+} A_{L}^{-}}^{-}(t)\left(1-P_{I_{L}^{+} A_{L}^{-}}\right) F_{I_{L}^{-} A_{L}^{+}}^{-}(t)\left(1-P_{I_{L}^{-} A_{L}^{+}}\right)\right]}{\log \left[F_{I_{L}^{-} A_{L}^{-}}^{-}(t)\left(1-P_{I_{L}^{-} A_{L}^{-}}\right)\right]}
\end{aligned}
$$

where each $F$. $(t)$ is the cumulative RT distribution function to the corresponding pair type and each $P$. is the probability of giving a positive response to pairs of that type.

Although the assessment function has a complicated form, it asks a simple question: When item and associative information are congruent, does this aid processing more (assessment greater than one) or less (assessment less than one) than would be expected if the two kinds of information were retrieved separately in parallel and did not interact? The numerator represents the amount of work needed for a participant to make a correct response to a congruent pair by time $t$ under the assumption that they always treated the two information sources separately and processed them in parallel, making a response (either positive or negative) on the basis of whichever is retrieved first. The denominator reflects the observed work done by time $t$ to make a correct response when item and associative information are actually congruent. If this ratio is greater than one, congruent processing is more efficient (i.e., yields higher accuracy in less time) than would be expected from parallel independent processing. If the assessment function is less than one, congruent processing is less efficient than would be expected from parallel independent processing, implying a limited ability to combine congruent item and associative information.

Although we refer the reader to Townsend \& Altieri (2012) and Altieri et al. (2014) for in-depth discussions of the assessment function, we make some remarks here that will hopefully aid the reader's intuition. If the assessment function equals one throughout, then item and associative information are processed independently, in parallel, with a self-terminating stopping rule-this follows from the definition of the function, as described above. If processing is extremely error-prone, however, this low accuracy can result in an assessment function less than one even under independent parallel self-terminating processing. Serial self-terminating processing can lead to assessment functions that deviate from one if the order in which item and associative information are retrieved allows decisions to be made on the basis of the first retrieved piece of information (e.g., if item information tends to be retrieved earlier, one would not need to retrieve associative information to reject a $I^{-} A^{-}$pair). Facilitatory and/or inhibitory interactions can yield assessment functions that are either greater or less than one depending on the degree to which processing is error-prone; interactions can not only enhance information leading to a correct response (assessment greater than one), they may also amplify noise (assessment less than one). Finally, we remark that exhaus- 


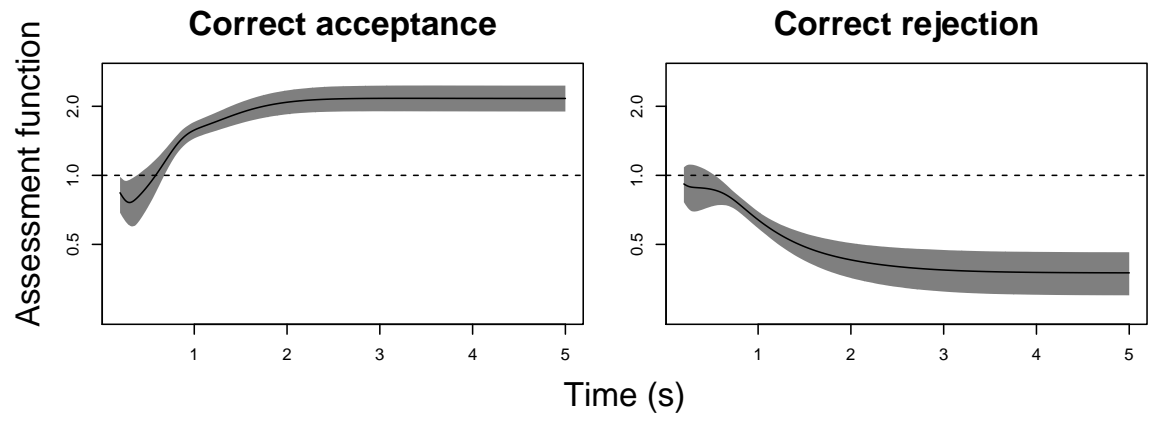

Figure 5: Capacity assessment functions for correct responses. Solid lines are posterior means while shaded regions are $95 \%$ credible intervals.

tive decision rules, regardless of the other aspects of the retrieval architecture, often lead to assessment functions that are greater than one, owing to the fact that exhaustive processing is exceptionally inefficient in incongruent situations.

As with the SIC, we compute each sample $n=1 . .10,000$ of each assessment function using the estimated RT distributions and response probabilities on that sample. Plugging these into the equations above (and dropping the $[n]$ index for clarity), each sample of the assessment function is given by

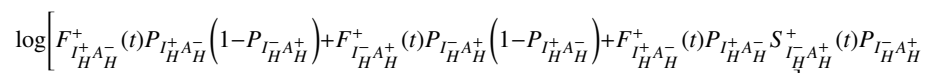

$$
\begin{aligned}
& A_{C A}[n](t)=\frac{\left.+S_{I_{H}^{+} A_{H}^{-}}^{+}(t) P_{I_{H}^{+} A_{H}^{-}} F_{I_{H}^{-} A_{H}^{+}}^{+}(t) P_{I_{H}^{-} A_{H}^{+}}+F_{I_{H}^{+} A_{H}^{-}}^{+}(t) P_{I_{H}^{+} A_{H}^{-}} F_{I_{H}^{-} A_{H}^{+}}^{+}(t) P_{I_{H}^{-} A_{H}^{+}}\right]}{\log \left[F_{I_{H}^{+}}^{+} A_{H}^{+}(t) P_{I_{H}^{+} A_{H}^{+}}\right]} \\
& \log \left[F_{I_{L}^{+} A_{L}^{-}}^{-}(t)\left(1-P_{I_{L}^{+} A_{L}^{-}}\right) P_{I_{L}^{-} A_{L}^{+}}+F_{I_{L}^{-} A_{L}^{+}}^{-}(t)\left(1-P_{I_{L}^{-} A_{L}^{+}}\right) P_{I_{L}^{+} A_{L}^{-}}+F_{I_{L}^{+} A_{L}^{-}}^{-}(t)\left(1-P_{I_{L}^{+} A_{L}^{-}}\right) S_{I_{L}^{-} A_{L}^{+}}^{-}(t)\left(1-P_{I_{L}^{-} A_{L}^{+}}\right)\right. \\
& A_{C R}[n](t)=\frac{\left.+S_{L_{L}^{+} A_{L}^{-}}^{-}(t)\left(1-P_{I_{L}^{+} A_{L}^{A}}\right) F_{I_{L}^{-} A_{L}^{-}}^{(t)}\left(1-P_{I_{L}^{-} A_{L}^{+}}\right)+F_{I_{L}^{+} A_{L}^{-}}^{-}(t)\left(1-P_{I_{L}^{+} A_{L}^{-}}\right) F_{I_{L}^{-} A_{L}^{+}}(t)\left(1-P_{I_{L}^{-} A_{L}^{+}}\right)\right]}{\log \left[F_{I_{L}^{-} A_{L}^{-}}^{-}(t)\left(1-P_{I_{L}^{-} A_{L}^{-}}\right)\right]}
\end{aligned}
$$

where $F_{. .}^{+}(t)$ and $F_{. .}^{-}(t)$ are the CDF's for response times when a positive or negative response is given in that condition, $S_{. .}^{+}(t)$ and $S_{. .}^{-}(t)$ are the survivor functions (one minus the $\mathrm{CDF}$ ) for response times when a positive or negative response is given in that condition, and $P$.. is the probability of giving a positive response in that condition.

Once again, different processes make different predictions about the form that the assessment function can take, as shown in Figure $3 \mathrm{C}$. After an initial period in which both observed assessment functions begin near one, the assessment function for correct acceptance responses is credibly greater than one, while the opposite is true for correct rejections (Figure 5). In answer to question 2, when both item and associative information match the contents of memory, processing is particularly efficient, more so than would be expected if the two kinds of information were processed independently in parallel. The same is not true for mismatching item and associative information- 
Table 5: Qualitative properties of SFT measures, indicated whether positive (Pos.) and/or negative (Neg.) deflections are allowed by a particular retrieval architecture. Bold text indicates that the observed function is consistent with what is allowed. Asterisks for independent serial exhaustive and coactive processing refer to the fact that they allow positive deflections in the SIC only if there is a corresponding negative deflection (and vice versa for independent serial exhaustive processing).

\begin{tabular}{|c|c|c|c|c|c|c|c|c|c|}
\hline \multirow{3}{*}{$\begin{array}{l}\text { Retrieval processes } \\
\text { Correct acceptance }\end{array}$} & \multirow[b]{3}{*}{ Correct rejection } & \multicolumn{4}{|l|}{ SIC } & \multicolumn{4}{|c|}{ Assessment } \\
\hline & & \multicolumn{2}{|c|}{ Correct acceptance } & \multicolumn{2}{|c|}{ Correct rejection } & \multicolumn{2}{|c|}{ Correct acceptance } & \multicolumn{2}{|c|}{ Correct rejection } \\
\hline & & Pos. & Neg. & Pos. & Neg. & Pos. & Neg. & Pos. & Neg. \\
\hline $\begin{array}{l}\text { Independent serial } \\
\text { self-terminating }\end{array}$ & $\begin{array}{l}\text { Independent serial } \\
\text { exhaustive }\end{array}$ & Yes & Yes & Yes* & Yes* & Yes & Yes & Yes & No \\
\hline $\begin{array}{l}\text { Independent serial } \\
\text { exhaustive }\end{array}$ & $\begin{array}{l}\text { Independent serial } \\
\text { self-terminating }\end{array}$ & Yes* & Yes* & Yes & Yes & Yes & No & Yes & Yes \\
\hline $\begin{array}{l}\text { Independent parallel } \\
\text { self-terminating }\end{array}$ & $\begin{array}{l}\text { Independent parallel } \\
\text { exhaustive }\end{array}$ & Yes & Yes & No & Yes & No & Yes & Yes & No \\
\hline $\begin{array}{l}\text { Independent parallel } \\
\text { exhaustive }\end{array}$ & $\begin{array}{l}\text { Independent parallel } \\
\text { self-terminating }\end{array}$ & No & Yes & Yes & Yes & Yes & No & No & Yes \\
\hline $\begin{array}{l}\text { Facilitatory } \\
\text { self-terminating }\end{array}$ & $\begin{array}{l}\text { Inhibitory } \\
\text { exhaustive }\end{array}$ & Yes & Yes & No & Yes & Yes & Yes & Yes & No \\
\hline $\begin{array}{l}\text { Facilitatory } \\
\text { exhaustive }\end{array}$ & $\begin{array}{l}\text { Inhibitory } \\
\text { self-terminating }\end{array}$ & Yes & No & Yes & Yes & Yes & Yes & Yes & Yes \\
\hline $\begin{array}{l}\text { Inhibitory } \\
\text { self-terminating }\end{array}$ & $\begin{array}{l}\text { Facilitatory } \\
\text { exhaustive }\end{array}$ & Yes & Yes & Yes & No & Yes & Yes & Yes & Yes \\
\hline $\begin{array}{l}\text { Inhibitory } \\
\text { exhaustive }\end{array}$ & $\begin{array}{l}\text { Facilitatory } \\
\text { self-terminating }\end{array}$ & No & Yes & Yes & Yes & Yes & No & Yes & Yes \\
\hline Coactive & Coactive & Yes* & Yes & Yes* & Yes & Yes & Yes & Yes & Yes \\
\hline
\end{tabular}

mismatches are processed less efficiently than independent parallel processing. Overall, it appears that mismatch information is inhibited whereas match information is mutually facilitatory.

\subsubsection{Conclusions from SFT}

Participants can only make positive or negative responses, which are the converse of each other. Whenever positive responses are self-terminating, negative responses must be exhaustive, and vice versa; facilitation of positive responses is inhibition for negative responses, and vice versa. Therefore, there are nine possible combinations of retrieval processes, as shown in Table 5. By comparing the qualitative properties of the observed SIC and assessment functions with those predicted by each type of processing, we find that the only set of retrieval processes that is fully consistent with the observed SIC and assessment functions is that in which positive responses arise from facilitatory exhaustive retrieval of item and associative information 4 . In other words, match information is mutually reinforced between item and associative retrieval while mismatch information arises separately from these two sources and races against the pooled match evidence, enabling negative responses to be made on the basis of either type of mismatch, as depicted in Figure 8

\footnotetext{
${ }^{4}$ SIC's for error responses are also consistent with this type of processing; see Appendix B
} 


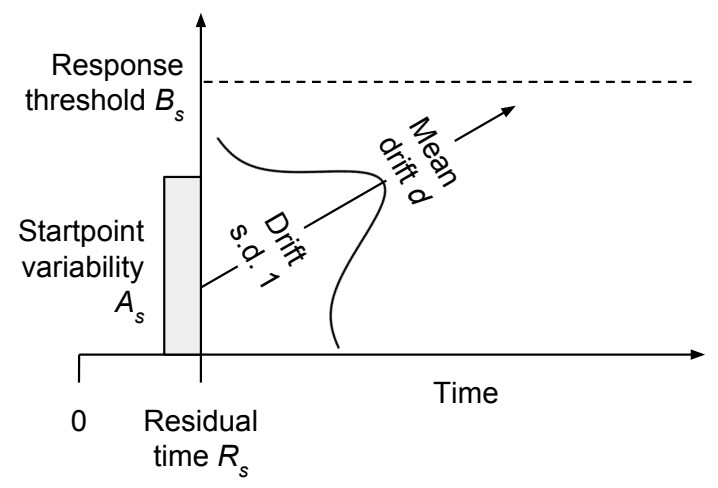

Figure 6: Diagram depicting a single linear ballistic accumulator (LBA) and associated parameters.

\subsection{Individual modeling}

While the description afforded by SFT has the virtue of being derived from purely qualitative distinctions between broad model classes, this analysis is limited by its reliance on group distributions which could obscure variability between individuals. In addition, while SFT provides strong evidence of interactions between item and associative information, it cannot easily answer question $1 \mathrm{~b}$, namely, whether interactions occur during retrieval or are a byproduct of how item and associative information are stored in memory, or both. To address these issues and answer this outstanding question, we augment our SFT analysis by jointly modeling accuracy and response time for each individual within a Linear Ballistic Accumulator (LBA) framework (Brown \& Heathcote 2008). LBA models have been useful for corroborating conclusions derived from SFT (Eidels et al., 2010, Donkin et al., 2014) and have the advantage of jointly modeling both accuracy and response time for both correct and error responses 5 In addition, the LBA modeling framework allows a natural way to express each of the seven possible parallel retrieval architectures and to separately estimate the contributions of interactions prior to retrieval versus those during retrieval.

\subsubsection{Linear ballistic accumulator models}

An LBA models decision making by assuming that evidence begins at a value sampled at uniform between zero and $A_{s}$, where $A_{s}$ varies for each participant $s$, as shown in Figure 6. Evidence then accumulates at a fixed rate $v$ sampled from a normal distribution on each trial with variance 1 ; the match evidence distribution has mean $d_{i j s}^{+}$, with the mean depending on item strength $i$, associative strength $j$, and subject $s$, while mismatch evidence has mean $d_{i j s}^{-}$. The LBA finishes when (if) the total amount

\footnotetext{
${ }^{5}$ While this is also a feature of our hierarchical Bayesian approach to estimate RT distributions, the LBA is much more strongly constrained in this respect.

${ }^{6}$ The LBA does not readily permit serial models, however our SFT results already argue against the two forms of independent serial processing.
} 
of accumulated evidence reaches a threshold $-B_{s}^{+}$for a match accumulator or $B_{s}^{-}$for mismatch—for each participant $s$.

We assume that up to four LBA's operate on each trial corresponding to item match, item mismatch, associative match, and associative mismatch information. These four accumulators are, respectively, associated with finishing-time density functions $f_{I}^{+}(t)$, $f_{I}^{-}(t), f_{A}^{+}(t)$, and $f_{A}^{-}(t)$ (distribution functions are denoted with capital $F$ 's). To properly identify the model, we assume the evidence for the two match accumulators is sampled from the same distribution (with mean $d_{i j s}^{+}$), similarly for the two mismatch accumulators (with mean $d_{i j s}^{-}$). Both match accumulators have the same threshold $B_{s}^{+}$ and both mismatch accumulators have the same threshold $B_{s}^{-}$while all accumulators have the same start variability parameter $A_{s}$ and residual non-decision time $R_{s}$. Finally, we allow that some trials might be contaminants with probability $c_{s}$; on a contaminant trial, a participant gives a positive response with probability 0.25 (i.e., the true $I^{+} A^{+}$ base rate) at a uniform time sampled between 0.2 and 5 seconds.

As in our SFT analyses, different retrieval architectures entail that different accumulators interact. If two accumulators interact during retrieval, their corresponding parameters are pooled via addition. For example, if the two match accumulators interact, the starting level of the pooled accumulator is sampled at uniform between zero and $2 A_{s}$, the evidence rate is sampled from a distribution with mean $2 d_{i j s}^{+}$and variance 2 , and accumulation terminates when evidence reaches $2 B_{s}^{+}$. To designate the pooled match accumulator, we drop the $I$ and $A$ subscripts, i.e., $f^{+}(t)$ is its finishing-time density. In this way, we can specify the likelihood of a "yes" response at time $t$ for each type of parallel retrieval architecture:

$$
\begin{aligned}
\left.L_{\mathrm{PST}} \text { (“yes", } t\right) & =\left\{f_{I}^{+}(t)\left[1-F_{A}^{+}(t)\right]+f_{A}^{+}(t)\left[1-F_{I}^{+}(t)\right]\right\}\left[1-F_{I}^{-}(t) F_{A}^{-}(t)\right] \\
\left.L_{\mathrm{PEX}} \text { (“yes", } t\right) & =\left[f_{I}^{+}(t) F_{A}^{+}(t)+f_{A}^{+}(t) F_{I}^{+}(t)\right]\left[1-F_{I}^{-}(t)\right]\left[1-F_{A}^{-}(t)\right] \\
\left.L_{\mathrm{FEX}} \text { ("yes", } t\right) & =f^{+}(t)\left[1-F_{I}^{-}(t)\right]\left[1-F_{A}^{-}(t)\right] \\
\left.L_{\mathrm{PST}} \text { (“yes", } t\right) & =f^{+}(t)\left[1-F_{I}^{-}(t) F_{A}^{-}(t)\right] \\
\left.L_{\mathrm{IEX}} \text { ("yes", } t\right) & =\left[f_{I}^{+}(t) F_{A}^{+}(t)+f_{A}^{+}(t) F_{I}^{+}(t)\right]\left[1-F^{-}(t)\right] \\
\left.L_{\mathrm{IST}} \text { ("yes", } t\right) & =\left\{f_{I}^{+}(t)\left[1-F_{A}^{+}(t)\right]+f_{A}^{+}(t)\left[1-F_{I}^{+}(t)\right]\right\}\left[1-F^{-}(t)\right] \\
\left.L_{\mathrm{COA}} \text { ("yes", } t\right) & =f^{+}(t)\left[1-F^{-}(t)\right]
\end{aligned}
$$

where "COA" is short for "coactive", and for the other models, the first letter denotes independent parallel ("P"), facilitatory ("F"), or inhibitory ("I") and the next two letters give the stopping rule for "yes" responses ("ST" for self-terminating, "EX" for exhaustive). Likelihoods for negative responses are obtained by switching the ${ }^{+}$and - superscripts and the stopping rule (if "yes" responses are self-terminating, "no" responses must be exhaustive and vice versa). We estimated each model parameterincluding which retrieval architecture was used - for each individual according to the hierarchical Bayesian model described in Appendix D The model was implemented in JAGS (Plummer. 2013), which was used to obtain 10,000 posterior samples split over 10 parallel chains after 2000 iterations of "burn-in" each.

Our subsequent analyses focus on the drift rates and retrieval architectures inferred using this model, since these parameters are those that directly address questions of 
Table 6: Posterior modes and 95\% credible intervals (in parentheses) of the quantity of retrieved evidence as assessed by the sum of the match and mismatch LBA drift rates. Shaded cells indicate intact $I^{+} A^{+}$pairs, which should be given a positive response.

\begin{tabular}{|c|c|c|c|c|c|}
\hline \multirow[b]{6}{*}{ हี } & & \multicolumn{4}{|c|}{ Associative strength } \\
\hline & & $A_{H}^{+}$ & $A_{L}^{+}$ & $A_{H}^{-}$ & $A_{L}^{-}$ \\
\hline & $I_{H}^{+}$ & $4.10(3.86,4.36)$ & $4.01(3.75,4.24)$ & $3.66(3.43,3.88)$ & $3.62(3.40,3.85)$ \\
\hline & $I_{L}^{+}$ & $4.25(3.99,4.50)$ & $4.16(3.89,4.38)$ & $3.63(3.41,3.86)$ & $3.63(3.41,3.87)$ \\
\hline & $I_{H}^{-}$ & $3.65(3.41,3.87)$ & $3.76(3.51,3.98)$ & $3.66(3.44,3.90)$ & $3.61(3.39,3.87)$ \\
\hline & $I_{L}^{-}$ & $3.75(3.52,3.98)$ & $3.85(3.60,4.07)$ & $3.71(3.49,3.96)$ & $3.69(3.48,3.96)$ \\
\hline
\end{tabular}

Table 7: Posterior modes and 95\% credible intervals (in parentheses) of the quality of retrieved memory evidence as assessed by the difference between the LBA drift rate for positive (match) accumulators and negative (mismatch) accumulators. Thus, values greater than zero indicate support for a positive response while those less than zero indicate support for a negative response. Shaded cells indicate intact $I^{+} A^{+}$pairs, which should be given a positive response.

\begin{tabular}{|c|c|c|c|c|}
\hline \multirow{6}{*}{$\begin{array}{l}\text { D. } \\
\text { है } \\
\text { है }\end{array}$} & \multicolumn{4}{|c|}{ Associative strength } \\
\hline & $A_{H}^{+}$ & $A_{L}^{+}$ & $A_{H}^{-}$ & $A_{L}^{-}$ \\
\hline & $1.60(1.40,1.77)$ & $0.72(0.61,0.86)$ & $0.11(0.00,0.23)$ & $-0.02(-0.14,0.084)$ \\
\hline & $1.51(1.35,1.66)$ & $0.36(0.24,0.49)$ & $-0.11(-0.22,0.01)$ & $-0.58(-0.70,-0.46)$ \\
\hline & $-0.30(-0.43,-0.19)$ & $-0.64(-0.77,-0.52)$ & $-0.91(-1.05,-0.78)$ & $-0.90(-1.03,-0.77)$ \\
\hline & $-0.31(-0.42,-0.18)$ & $-0.59(-0.72,-0.47)$ & $-0.99(-1.13,-0.86)$ & $-1.11(-1.26,-0.98)$ \\
\hline
\end{tabular}

theoretical interest. For completeness, however, we report estimates of the other model parameters (boundary separation, start-point variability, etc.) in Table D.1.

\subsubsection{Interactions prior to retrieval}

It is possible that item and associative information could be encoded or stored in such a way that they are correlated, irrespective of the retrieval architecture. For example, if strongly encoded item information makes it easier to encode associative information (or vice versa), this would constitute a facilitatory interaction at storage that would be separate from any facilitation that might occur as a function of the architecture of the retrieval processes. Eidels et al. (2011) termed this a distinction between "pre-accumulator" and "post-accumulator" interactions, where "pre-accumulator" in our case applies to processes like storage that occur prior to retrieval while "postaccumulator" applies to the retrieval processes themselves. These two kinds of interaction are explicitly disentangled within our LBA framework, which separates retrieval architecture-_"post-accumulator" interactions that result from pooling information across accumulators-from the inputs to that architecture- the drift rates that reflect the evidence retrieved from memory as well as any "pre-accumulator" interactions. Therefore, in this section we examine the posterior distributions of drift rates to assess the evidence for interactions prior to retrieval. In the next section, we examine the posterior probabilities assigned to each retrieval architecture, which provides evidence for interactions during retrieval.

The drift rates that serve as inputs to the retrieval process can be characterized in terms of the total quantity of memory evidence as well as the quality of that evidence. For each combination of item strength $i$, associative strength $j$, and subject $s$, the overall quantity of retrieved evidence is represented by the sum of the drift rates for the positive (match) and negative (mismatch) accumulators: $d_{i j s}^{+}+d_{i j s}^{-}$. The higher this quantity, 
Table 8: Posterior modes and $95 \%$ credible intervals (in parentheses) of the interactions between item and associative strength on total drift rates, i.e., the total quantity of retrieved memory evidence. Bold text indicates that the $95 \%$ credible interval excludes zero and shaded cells indicate intact $I^{+} A^{+}$pairs, which should be given a positive response.

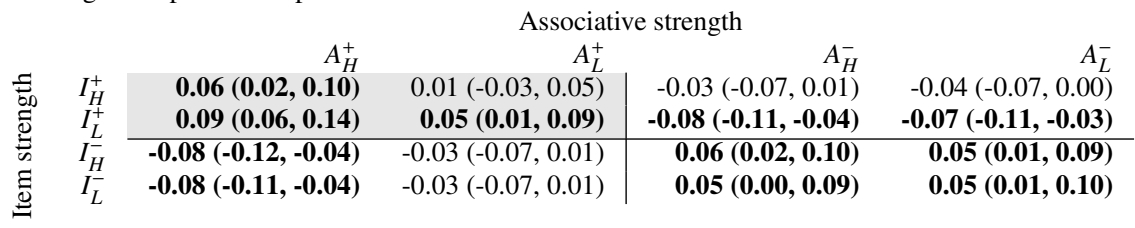

Table 9: Posterior modes and $95 \%$ credible intervals (in parentheses) of the interactions between item and associative strength on retrieved memory evidence, i.e., the difference between positive and negative drift rates. Bold text indicates that the $95 \%$ credible interval excludes zero and shaded cells indicate intact $I^{+} A^{+}$ pairs, which should be given a positive response.

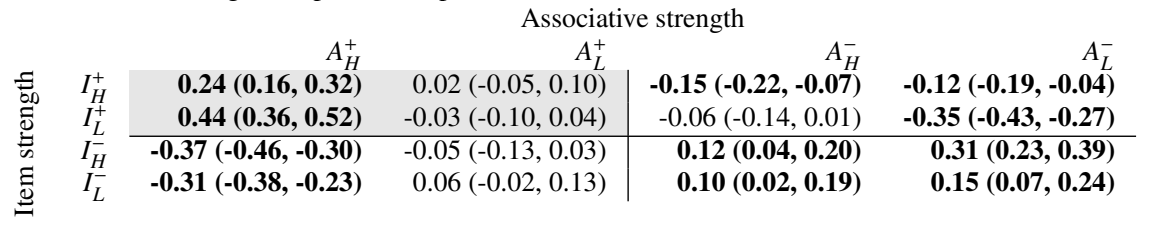

the posterior distributions of which are given in Table 6 , the more retrieved memory evidence is flowing into the system. The difference in drift rates reflects the quality of that evidence. By analogy with $d^{\prime}$ in signal detection, we quantify the retrieved memory evidence as the degree to which retrieval favors a positive versus negative recognition response, i.e., $d_{i j s}^{+}-d_{i j s}^{-}$. To the extent that this quantity is greater than zero, memory evidence favors a positive response, and to the extent that it is less than zero, memory evidence favors a negative response. The posterior distributions of average retrieved memory evidence for each pair are given in Table 7 .

As shown in Table 6, the total quantity of retrieved evidence is generally greater for $I^{+} A^{+}$pairs than for other pair types which all have comparable levels of total input (compare the upper left quadrant of Table 6 to the other three quadrants). This suggests the presence of an interaction, in the sense that the total input to retrieval is not an additive function of the strengths of the item and associative information in a pair. As in analysis of variance, we can compute the average degree to which item and associative strength interact to affect total retrieved evidence as:

$$
\operatorname{Mean}_{s}\left[d_{i j s}^{+}+d_{i j s}^{-}\right]-\operatorname{Mean}_{i j s}\left[d_{i j s}^{+}+d_{i j s}^{-}\right]-\operatorname{Mean}_{i s}\left[d_{i j s}^{+}+d_{i j s}^{-}\right]-\operatorname{Mean}_{j s}\left[d_{i j s}^{+}+d_{i j s}^{-}\right],
$$

i.e., the average deviation in summed drift rates from what would be expected if they were a purely additive function of item strength $i$ and associative strength $j$. As shown in Table 8, there are several credible deviations from additivity: Total retrieved evidence is greater than would be expected from additivity when item and associative information agree $\left(I^{+} A^{+}\right.$and $\left.I^{-} A^{-}\right)$and is less when they disagree $\left(I^{+} A^{-}\right.$and $\left.I^{-} A^{+}\right)$.

We can ask the same question regarding the quality of retrieved memory evidence: The average degree to which item and associative strength interact to affect evidence 
quality is given by

$$
\operatorname{Mean}_{s}\left[d_{i j s}^{+}-d_{i j s}^{-}\right]-\operatorname{Mean}_{i j s}\left[d_{i j s}^{+}-d_{i j s}^{-}\right]-\operatorname{Mean}_{i s}\left[d_{i j s}^{+}-d_{i j s}^{-}\right]-\operatorname{Mean}_{j s}\left[d_{i j s}^{+}-d_{i j s}^{-}\right],
$$

i.e., the average deviation in drift differences from what would be expected if drift differences were a purely additive function of item strength $i$ and associative strength $j$. As shown in Table 9, retrieved evidence is higher than expected from additivity for $I^{+} A^{+}, I^{+} A^{-}$, and $I^{-} A^{+}$pairs but lower than expected for $I^{-} A^{-}$pairs (recall that lower numbers indicate greater support for a negative response and higher numbers indicate greater support for a positive response, which is an error for $I^{-} A^{-}$pairs).

These analyses thus provide some evidence that item and associative information interact prior to retrieval, as reflected in the inputs to the retrieval architecture as modeled within the LBA framework. These interactions could, in part, reflect our experimental design, which entails repeating whole pairs to increase associative strength. Such repetitions are likely to increase the strength of the component items as well; this is evident above, in that for $I^{+} A^{+}$pairs, the quality of retrieved memory evidence is particularly enhanced by repeating whole pairs $\left(A_{H}^{+}\right.$versus $\left.A_{L}^{+}\right)$. There is no sign, however, that repeating the components of a pair separately (presenting AA or BB for $I_{H}^{+}$pairs) interferes with whole pair (AB) associations. Such interference could manifest in two ways that would reflect either diminished speed or accuracy for $I_{H}^{+}$pairs: first, as a negative interaction for $I_{H}^{+} A_{L}^{+}$or $I_{H}^{+} A_{H}^{+}$pairs in either retrieval speed or memory evidence, neither of which we found; second, as a negative retrieval speed interaction for $I_{H}^{+} A_{L}^{-}$or $I_{H}^{+} A_{H}^{-}$pairs or positive memory evidence interaction for those same pair types, neither of which we found (recall that positive memory evidence would lead one to falsely recognize an $I^{+} A^{-}$pair). We note, however, that form of these interaction analyses depends on the assumption that memory evidence can be characterized as Gaussian distributions on an interval scale. Although this assumption yields a good account of our data (Figure D.2), it is logically possible to allow more esoteric distributions (e.g., Jones \& Dzhafarov 2014) which would entail different ways of measuring the presence of drift rate interactions. Finally, we note that because all retrieval architectures take the same drift rates as inputs, the above analyses are agnostic with respect to how these inputs subsequently interact during retrieval.

\subsubsection{Interactions during retrieval}

Even allowing for potential interactions prior to retrieval, there remains overwhelming evidence for interactions during retrieval. Retrieval interactions are indicated by the processing architecture estimated for each participant. The posterior probability that a participant is best described by a particular LBA retrieval architecture is shown in Figure 7. with the vast majority of participants (112 out of 118) best described by facilitatory exhaustive processing for positive responses (with corresponding inhibitory selfterminating processing for negative responses) - the same model supported by SFT. Additionally, we demonstrate in Appendix D that interactions prior to and during retrieval do not trade-off with one another and reflect independent contributions to the retrieval processes modeled by the LBA. Thus, in answer to question $1 \mathrm{~b}$, while it is possible that interactions occur between item and associative information prior to retrieval, it is also the case that item and associative memory interact during retrieval. 


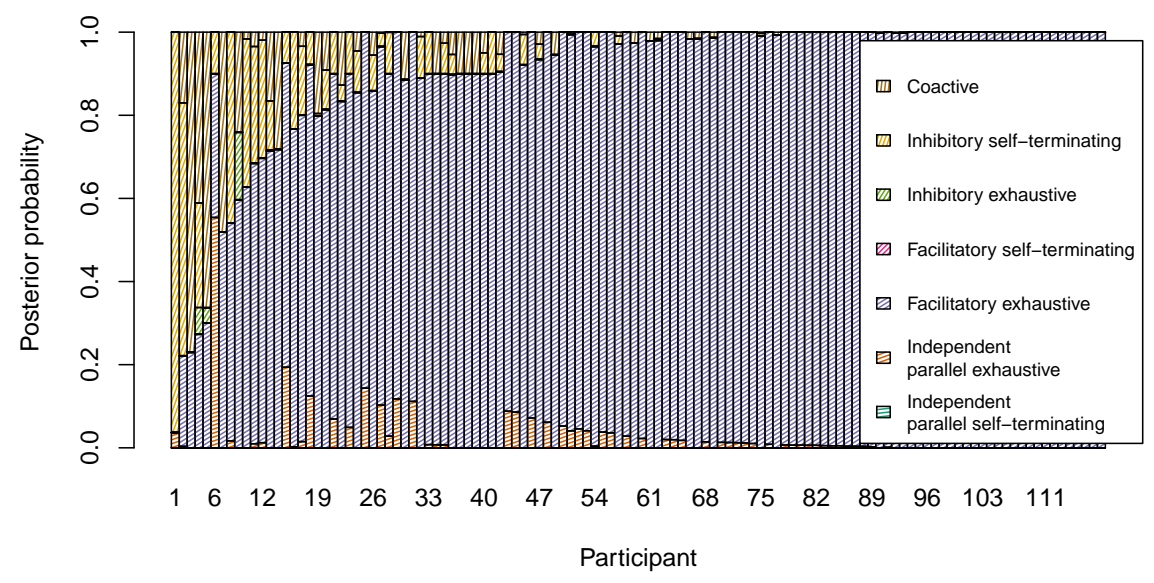

Figure 7: Posterior probability that each participant uses a particular retrieval architecture. Labels are with respect to the processes leading to positive responses.

These results are a strong validation of the conclusions drawn from SFT and imply that facilitatory retrieval of item and associative information is a relatively ubiquitous characteristic of human memory.

\section{Discussion}

Item and associative information are retrieved concurrently, with positive memory evidence arising from a pooled match between the item and associative information within a test pair. This conclusion was based on model-independent qualitative properties of response dynamics, as measured by tools from SFT (Townsend \& Nozawa, 1995: Townsend \& Altieri, 2012), and was confirmed by quantitative modeling of individuals. This effort not only yielded important results about human memory, it also demonstrated how SFT can answer important psychological questions in domains where it has previously been difficult to apply, particularly when coupled with Bayesian estimation of RT distributions (for further developments along these lines, see Houpt et al. 2016). After summarizing the final supported model of item and associative retrieval, we delve more deeply into the potential generality of this model and the broader implications of the model and the methods we used to identify it.

\subsection{Final supported model}

Considering the literature and the results presented here, we are left with a wellconstrained account of item and associative memory, depicted schematically in Figure 8 Item and associative information are represented in a separable fashion, either in separate memory stores or as separate features of a single memory trace. Although item information may be available earlier than associative information, both kinds of information are eventually retrieved simultaneously and interact with one another. The 


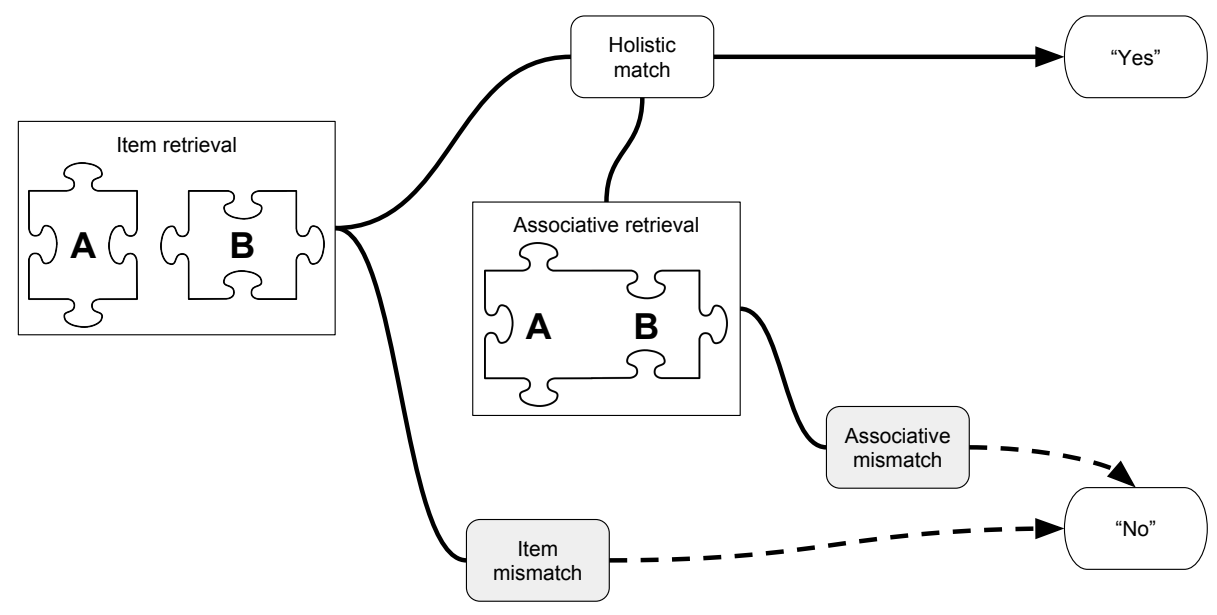

Figure 8: A schematic description of the architecture by which item (separate pieces "A" and "B") and associative information (joined piece "AB") are retrieved. Item and associative information that match the contents of memory are pooled into a holistic match that is used as the basis for positive recognition decision ("yes" responses) while mismatching information is processed separately for the two sources, with either kind of mismatch capable of resulting in a negative recognition decisions ("no" responses).

degree of match is based on an interactive, holistic comparison of both item and associative features to memory while the degree of mismatch is based on separate assessments of item and associative mismatch. These two kinds of mismatch information accumulate in parallel with the pooled match and the final decision (for the decision rule needed in the present paradigm) is based on whichever of these processes finishes first. In the remainder of the Discussion, we consider the generality of this account, how it meshes with extant results, and how it can help guide further empirical work and theoretical developments.

\subsection{Generality}

Our goal is to constrain theories of memory in general, rather than theories of memory for just particular classes of stimuli or particular tasks. Although we found broad support for a model in which matching item and associative information are processed holistically while mismatching information is processed separately, we must consider the generality of these results and the methods used to reach them.

\subsubsection{Stimuli}

Memory for items and associations is often studied using verbal materials, so is it possible that different processes underlie memory for words than memory for images? One potential difference between images and words is that while participants have usually encountered stimulus words prior to the experiment, they would not have seen any of our stimulus images before; as a result, context could be a more important cue for retrieval of verbal stimuli as opposed to images. However, because our images were all commonplace scenes, participants could be expected to have encountered the content of each image (e.g., trees, clouds, cars, buildings, furniture, etc.) prior to the experiment, 
necessitating a role for episodic context during recognition. Furthermore, the contexts in which participants typically experience either verbal or image content is as part of meaningful continuous interactions with text, speech, objects, and scenes, in contrast to the disconnected, punctate manner with which such stimuli are presented within a typical memory experiment (Cox \& Shiffrin in press). As a result, the experimental episodic context can be expected to be equally distinct from typical pre-experimental experience for both classes of stimuli.

Even if memory for individual words and images are not qualitatively different, it may be that associations between words are encoded in an explicit conceptual manner (e.g., by imagining a sentence connecting the two words) whereas image associations may be stored differently, perhaps as another image. Our design precludes the possibility that image pairs can be easily encoded as just another image, since the two images in a pair were assigned different random vertical offsets on each presentation (e.g., between study and test). If participants were to try to encode the image pair as a larger image, they would have to imagine aligning the two halves themselves, in essence creating a new "mental image". Such mental imagery has been found to be a strong predictor of memory for words and word pairs as well (Paivio, 1969, Bower, 1970, McGee, 1980), suggesting that word and image associations, to the extent that they are encoded correctly, may not be so different after all. Finally, if image associations were processed as just another, albeit larger, image, there would be no reason for mismatching item and associative information to be processed separately.

Verbal stimuli would make it difficult to implement our full double factorial design. The difficulty lies in forming $I^{-} A^{+}$pairs, pairs that preserve associative information but disrupt item information. One possibility would be to use words with well-defined relations (e.g., foot-shoe) and replace them with words that share a similar relation (e.g., hand-glove), but this would seem to restrict the available stimuli to a fairly small set. A less restrictive approach would be to use random word pairs (e.g., baby-voyage) and replace them with synonyms (e.g., infant-journey) under the assumption that the overlapping semantic features between the component words would yield similar relational properties in each case. Although within-pair semantic similarity has been found to yield greater rates of both correct and false recognition of word pairs (Greene \& Tussing, 2001), it is not clear whether preserving only a portion of the semantic features of each word would be very effective in preserving any associative information. Images from the same scene preserve both low-level visual features (e.g., color distribution) and high-level semantics (scene content or "gist"; Andermane \& Bowers, 2015), whereas synonymous words tend to share only high-level features but have different low-level features (spelling/pronunciation). We have recently carried out experiments in our lab that study the extent to which event associations depend on low- or high-level features of both words and images, and consider this a productive avenue to travel.

\subsubsection{Tasks}

Given that item and associative information are retrieved in parallel, that they facilitate rather than compete with one another, and that interactions at retrieval occur regardless of potential correlations prior to retrieval (e.g., at storage), we expect our conclusions apply generally across a variety of memory tasks. Tasks that require retrieval of only one kind of information (e.g., pure item recognition or pure associative 
recognition) are special cases of our paradigm, and to the extent that task-irrelevant information is retrieved anyway, it will act to facilitate correct recognition (Tulving \& Thompson 1973). We do expect, however, that different tasks may entail different stopping rules. For example, in a dual-item recognition task where rearranged $I^{+} A^{-}$ pairs did not need to be rejected, we would expect that participants, to the extent that they follow instructions, will only use item mismatch to reject a pair while an associative match, if present, would continue to facilitate item recognition. Exactly this result has been found (Clark \& Shiffrin, 1987; Gronlund \& Ratcliff, 1989, Cohn \& Moscovitch 2007), even when associative encoding is discouraged (Jou, 2010). In tasks that require separate judgments for the item and associative content of a pair (Hockley \& Cristi 1996b; Buchler et al., 2008, 2011, participants could rely on the different mismatch signals to make their judgments, although the holistic match would continue to introduce a bias, exactly as has been reported.

One facet of memory that we did not explicitly investigate in this study was interference between competing entities in memory. Interference for items is typically studied by simply increasing the number of items stored in memory, which leads to moderate decreases in the ability to discriminate between studied and unstudied items (Murnane \& Shiffrin, 1991). For associations, interference is typically investigated in terms of "fan", i.e., the number of pairs that contain an overlapping element (Anderson 1983a), such that the ability to tell whether a pair is intact or rearranged is impaired when the pair contains an item that has appeared in many pairs during study (Wickelgren \& Corbett, 1977). While we found no evidence that self-pairs (AA and BB) interfered with whole pairs $(\mathrm{AB})$ in this way, we would expect that presenting overlapping pairs (e.g., $\mathrm{AC}, \mathrm{AD}$ ) would selectively interfere with the retrieval of associative mismatch information and leave item mismatch retrieval unaffected (assuming equal item frequency), a pattern that has been reported before using accuracy measures (Criss \& Shiffrin, 2004, 2005, Buchler et al., 2008, 2011, Aue et al., 2012). It is not immediately clear to us whether overlapping pairs would also interfere with the retrieval of a holistic match; since match information arises jointly from both item and associative sources, it seems likely that interfering associations could intrude on the holistic match, but additional study would be needed to decide this question.

Finally, we note that our focus was on the kinds of association stored via cooccurrence, which is necessarily episodic. Semantic associations may have different properties (Dosher. 1984), but as we argue below, learning such associations is usually predicated on the storage and retrieval of more basic event associations, and the interactions we found between item and associative retrieval can help us understand the relationship between episodic and semantic associations.

\subsubsection{Systems Factorial Technology}

Although our primary interest was to understand how item and associative information are retrieved from memory, pursuing this interest made extensive use of Systems Factorial Technology, even extending it via novel analyses (using Bayesian estimation of response time distribution) and to novel predictions regarding interactive processes. We expect that our experimental and analytical methods can be easily exported to other domains within and beyond the study of memory. Regarding predictions for interactive systems, while we were able to make some modest analytic predictions regarding 
interactive parallel systems, the complexity of such systems forced us to explore many of them via simulation, as described in Appendix A. Although these explorations were quite thorough, they cannot be as definitive as analytic proofs, which remain an active area of exploration. For example, while the analytic form of the SIC is known for some classes of coactive system (Townsend \& Nozawa, 1995, Fific et al., 2008, Houpt $\&$ Townsend, 2011), there as yet is no general proof that covers all coactive systems, let alone those with more complex interactions of the kind we considered. While the converging evidence from our various analyses and their relation to other results in the literature (as discussed both above and below) puts our conclusions regarding memory on firm footing, our efforts also contribute to the ongoing project to understand the qualitative signatures of different cognitive systems and to develop experimental means to detect them (Townsend \& Wenger, 2004; Eidels et al., 2011; Little et al., 2011; Yang et al. 2014).

\subsection{Implications}

We now consider some of the broader implications of the account of item and associative retrieval inferred from the present study.

\subsubsection{Are item and associative retrieval independent?}

We can definitively rule out independence of item and associative retrieval. The assumption of independence lies at the core of many popular views of item and associative retrieval, including the process dissociation procedure (Jacoby, 1991), mixture analysis of ROC curves (Yonelinas, 1997), and the analysis of additive ERP components (Rugg \& Curran, 2007). Because this assumption is violated, any conclusions drawn within those frameworks are invalid with respect to item and associative retrieval (see also Curran \& Hintzman, 1995; Hillstrom \& Logan, 1997). While it is logically possible for this lack of independence to arise from independent item and associative retrieval processes operating on strongly correlated memory representations, our individual modeling results render this interpretation untenable, since interactions at retrieval were strongly supported even in a model that allows correlations prior to retrieval.

Many of the above procedures-rendered invalid by the lack of item and associative independence- have been used to support a distinction between a "familiarity" process that retrieves item information and a "recollection" process that retrieves associative information. Although these processes were originally conceived as operating independently (Mandler, 1980), many modern applications of these terms have effectively abandoned that assumption, with associative information being available via both familiarity and recollection (Parks \& Yonelinas, 2015) and ERP signals thought to be pure reflections of familiarity and recollection actually supporting both item and associative retrieval (Speer \& Curran, 2007). Given that item and associative information are separable and yield different mismatch signals, our results are not inconsistent with studies that begin from the assumption of distinct familiarity and recollection processes. Rather, they provide a more theoretically coherent set of constructs with which to describe these prior results: to the extent that different behavioral or neural signals 
are found to be associated with item and associative information, they reflect the operation of one or the other mismatch accumulator and, potentially, different contributions to the holistic match signal.

\subsubsection{What is associative information?}

Throughout this research, we have identified associative information only in a functional sense, that is, associative information is whatever gets strengthened by repeating an intact pair during study (going from $A_{L}^{+}$to $A_{H}^{+}$) that is separate from what gets strengthened by repeating an item during study (going from $I_{L}^{+}$to $I_{H}^{+}$). Although it seems reasonable to assume that item information refers to the conceptual (and, perhaps, perceptual) content of the item event, it is less clear what associative information could be, independent of the items being associated. This kind of question has persisted in psychology for some time (Köhler, 1941, Asch, 1969), and requires us to reiterate that, although many theoretical constructs have been given the name "association", our focus is on associations in episodic memory, that is, the information that is encoded as a result of events occurring nearby in time and space.

Because mismatching associative information can be tracked separately from mismatching item information, the two kinds of information must be "separable" in some sense, even if they are not qualitatively dissimilar. Models of episodic memory offer many approaches toward representing associations in a form that is separable from items, such as links in a network (Anderson, 1983b; Gillund \& Shiffrin, 1984), concatenation of item features (Hintzman, 1988; Shiffrin \& Steyvers, 1997), features added to memory traces (Criss \& Shiffrin 2004; Cox \& Shiffrin in press), outer products (Humphreys et al., 1989), or convolutions (Murdock, 1982). These models offer little guidance, however, regarding how those representations actually enter into memory in the first place. Pre-existing semantic or similarity relations between items lead to stronger encoding of an association (Thomson \& Tulving, 1970, Dosher, 1984, Dosher \& Rosedale, 1991; Greene \& Tussing, 2001), and interference only occurs between pairs that are made of the same types of items (e.g., word-word versus word-face pairs; Criss \& Shiffrin, 2005), such that it is clear that associative information depends at least in part on the information contained in the associated items, especially relational features and features shared between items. While memory models of all stripes could easily implement this phenomenon, none of them predict it a priori.

One way by which associative information could be derived from items, while still remaining separable, is via unitization (Smith \& Haviland, 1972; Czerwinski et al., 1992; Shiffrin \& Lightfoot, 1997). Unitization refers to the creation of a novel entity that is "more than the sum of its parts", just as a word is more than a collection of letters or a painting more than a collection of brushstrokes, a topic often studied in Gestalt psychology (Wagemans et al. 2012a b) as well as in the study of the perception of both simple and complex stimuli (Townsend \& Wenger, 2015). The formation of unitized representations may be encouraged by semantic elaboration of either word pairs (Graf \& Schacter, 1985, Prior \& Bentin, 2003, 2008) or image pairs (Kan et al., 2011), resulting in the storage of stronger associative information. As in the present study, repetition of intact word pairs has been found to enhance memory for their association beyond any benefit from repeating the component items (Kilb \& Naveh-Benjamin, 2011, Parks \& Yonelinas, 2015), suggesting that associative information is a unitized representation 
of the complete pair that can be strengthened separately from representations of items. We will return to the possibility that associative information results from unitization below.

\subsubsection{What is associative retrieval?}

Although item and associative information are can be represented separately, the degree of match between item and associative information is pooled into a single holistic signal. For them to be combined this way means that item and associative retrieval processes must yield similar outputs. This is inconsistent with theories that posit qualitatively different mechanisms for item and associative retrieval, including most dual-process theories which assume either that item and associative retrieval are independent (via separate "familiarity" and "recollection" processes Yonelinas, 1997) or that associative retrieval can only serve to suppress an item match (via "recall-toreject"; Rotello \& Heit, 2000) rather than enhance it. At the same time, the ability for mismatch information to arise separately from item and associative retrieval is not easily accommodated by either single-process or summed dual-process models (Kelley $\&$ Wixted, 2001) which entail pooling of both match and mismatch information into a univariate "memory strength".

The apparent asymmetry between pooled match and separate mismatches is, however, consistent with functional accounts of the different subregions of the hippocampus (Kumaran \& Maguire, 2009) and with results in other memory domains in which match and mismatch information are tracked separately, such as short-term recognition (Mewhort \& Johns, 2000) and categorization (Tversky, 1977; Stewart \& Brown, 2005). It is also in line with likelihood-based models of memory which, in contrast to strength models, give different weight to match and mismatch information (Shiffrin \& Steyvers, 1997, McClelland \& Chappell, 1998).

Taken together, we hypothesize that associative retrieval occurs similarly to item retrieval in that it consists of comparing the associative information in the test pair to that stored in memory. In both cases, the output is a set of signals reflecting the degree to which each type of information matches or mismatches the contents of memory. Item and associative match information could be mutually facilitatory in two ways: first, by cross-talk between between two otherwise separate match accumulators (Townsend \& Wenger. 2004); second, by computing a match using a compound cue that combines both item and associative information and compares them jointly to memory (Ratcliff \& McKoon, 1988, Dosher \& Rosedale, 1989, 1997). In the first case, item and associative matches are still processed separately but, according to our results, these processes communicate so strongly with one another that they effectively act as a single process. In the second case, a single match process receives a single holistic input signal that jointly reflects item and associative match, a kind of partial coactivation (partial because only match, not mismatch, processes are combined). Unfortunately, it is unlikely that one can experimentally distinguish strongly-interactive-but-separate processes from a single holistic process, though this does not preclude testing specific implementations of these two types of facilitatory model (Colonius \& Townsend, 1997). One possible test relies on the fact that a holistic match can only yield a decision on the basis of combined evidence whereas separate channels can result in separate decisions; as a result, a holistic match should yield similar performance regardless of the 
decision rule whereas separate processes could yield different performance (Townsend $\&$ Wenger, 2004). The fact that associative information intrudes even when decisions need only be based on items (Tulving \& Thompson, 1973, Clark \& Shiffrin, 1987) and vice versa (Buchler et al., 2008) suggests that a holistic match is the more likely of these two candidates.

We address one final point demonstrated in prior studies of retrieval dynamics, namely, that associative retrieval is slower than item retrieval (Gronlund \& Ratcliff, 1989, Rotello \& Heit, 2000). From the viewpoint that associative information arises from the unitization of item information, it is clear that associative retrieval depends on having sufficient item information for a unit to be formed "on the fly" within the trial. Thus, associative retrieval must be slower than item retrieval, which is consistent with the use of early item information as a baseline or "gate" for later associative decisions, as mentioned in the Introduction (Hockley, 1991; Criss \& Shiffrin, 2005; Cox \& Shiffrin in press). These considerations also make it very likely that the form of parallel interactive processing that takes place between item and associative retrieval is a type of "continuous-flow" in which item and associative retrieval may begin at different times but then proceed concurrently. Although qualitative properties of many continuous-flow systems remain to be worked out (see Schweickert, 1989, Townsend \& Fikes, 1995, Liu, 1996), in general they represent a hybrid of serial and parallel processing and, as such, can demonstrate behavior characteristic of either type. For example, the cascade model, in which processing stages occur in a fixed order but overlap in time and operate in parallel (McClelland, 1979), can under certain conditions mimic the properties of a strictly serial model (Ashby. 1982). Because of their potential complexity, it is likely that empirical tests can only be made of specific continuous-flow models, rather than the general class (for an example of a continuous-flow model of associative recognition, developed prior to the work in this article, see Cox \& Shiffrin, in press).

\subsubsection{From episodic to semantic associations}

Over time, the ability to store and retrieve information about the co-occurrence of events underlies the learning of a great deal of semantic information, such as word learning (co-occurrence of words and referents; Smith \& Yu, 2008; Smith et al., 2014), causal learning (Zacks \& Tversky, 2001), and learning whole object representations (Czerwinski et al., 1992, Gauthier \& Tarr, 1997, Shiffrin \& Lightfoot, 1997; Nelson \& Shiffrin 2013). In a broad sense, most memory begins as "associative" in that events and objects are collections of isolated entities. Only as these collections are re-experienced many times do the entities merge to become "items" in their own right. This is precisely the process of unitization described above, which is why we consider it a natural description of associative information. This transition from associative to item information - with a concomitant transition from slow to fast retrieval as the unitized representation can be retrieved in toto rather than constructed "on the fly"-helps explain why well-learned semantic associations are retrieved more quickly than novel event associations (Dosher, 1984, Dosher \& Rosedale, 1991). Evidence for exactly this kind of representational transition has been found in the function of perirhinal cortex to encode novel unitized associations, in contrast to other regions which encode well-learned item information (Cowell et al., 2006; Haskins et al., 2008; Staresina \& 
Davachi, 2010; Cowell et al., 2010). In addition, the kind of facilitatory interactions we found between item and associative retrieval have been implicated in the learning of whole object and category representations (Goldstone, 2000).

\subsubsection{Associative deficits in aging}

Older adults have been found to show deficits for associative memory, relative to their memory for individual items (Naveh-Benjamin, 2000, Ratcliff et al., 2011; , McKoon \& Ratcliff, 2012). These deficits are reduced when the components of the association are already related, rather than representing a novel association (Naveh-Benjamin et al. 2003), consistent with our suggestion that associative information arises from a unitization process that transforms pairs into single items with repeated exposure. More pertinent to our results, however, is that the age-related associative deficit often manifests as an increased tendency to falsely recognize rearranged pairs rather than failure to recognize intact pairs (Old \& Naveh-Benjamin, 2008, Rhodes et al., 2008, NavehBenjamin et al. 2009). This suggests that the age-related associative deficit arises from a malfunction in the process we identified as accumulating associative mismatch information. The apparent ubiquity of this malfunction suggests it is not strategic, i.e., it is not merely that older adults "choose" not to engage this process, but we leave further investigation for future research.

\section{Conclusion}

The focus of the present work has been how information about individual events ("items") and combinations of events ("associations") are stored and retrieved from memory. Our conclusion, that item and associative information are stored in separable fashion and are retrieved concurrently with matching item and associative information pooled into a single holistic signal, was derived from careful study of the dynamics with which these kinds of information are retrieved. Although we have only begun to explore them, the implications of this conclusion are broad, owing to the fact that these processes are embedded in a memory system that supports cognition and action throughout a lifetime.

\section{Acknowledgments}

This work was supported by the National Science Foundation (grant number BCS0951612). We thank Bria Harris and Tommy Knoerl for their assistance with data collection.

Altieri, N., Townsend, J. T., \& Wenger, M. J. (2014). A measure for assessing the effects of audiovisual speech integration. Behavior Research Methods, 46, 406-415.

Andermane, N., \& Bowers, J. S. (2015). Detailed and gist-like visual memories are forgotten at similar rates over the course of a week. Psychonomic Bulletin $\mathcal{F}$ Review, 22, 1358-1363.

Anderson, J. R. (1983a). Retrieval of information from long-term memory. Science, 220, 25-30. 
Anderson, J. R. (1983b). A spreading activation theory of memory. Journal of Verbal Learning and Verbal Behavior, 22, 261-295.

Anderson, J. R., \& Bower, G. H. (1973). Human Associative Memory. Oxford, England: V. H. Winston \& Sons.

Asch, S. E. (1969). A reformulation of the problem of associations. American Psychologist, 24, 92-102.

Ashby, F. G. (1982). Deriving exact predictions from the cascade model. Psychological Review, 89, 599-607.

Aue, W. R., Criss, A. H., \& Fischetti, N. W. (2012). Associative information in memory: Evidence from cued recall. Journal of Memory and Language, 66, 109-122.

Aue, W. R., Criss, A. H., \& Novak, M. D. (2017). Evaluating mechanisms of proactive facilitation in cued recall. Journal of Memory and Language, 94, 103-118.

Bower, G. H. (1970). Imagery as a relational organizer in associative learning. Journal of Verbal Learning and Verbal Behavior, 9, 529-533.

Brown, S., \& Heathcote, A. (2008). The simplest complete model of choice response time: Linear ballistic accumulation. Cognitive Psychology, 57, 153-178.

Buchler, N. G., Faunce, P., Light, L. L., Gottfredson, N., \& Reder, L. M. (2011). Effects of repetition on associative recognition in young and older adults: Item and associative strengthening. Psychology and Aging, 26, 111-126.

Buchler, N. G., Light, L. L., \& Reder, L. M. (2008). Memory for items and associations: Distinct representations and processes in associative recognition. Journal of Memory and Language, 59, 183-199.

Clark, S. E., \& Shiffrin, R. M. (1987). Recognition of multiple-item probes. Memory $\mathcal{E}$ Cognition, 15, 367-378.

Cohn, M., \& Moscovitch, M. (2007). Dissociating measures of associative memory: Evidence and theoretical implications. Journal of Memory and Language, 57, 437454.

Colonius, H., \& Townsend, J. T. (1997). Activation-state representation of models for the redundant-signals-effect. In A. A. J. Marley (Ed.), Choice, Decision, and Measurement. NJ: Lawrence Erlbaum Associates.

Cowell, R. A., Bussey, T. J., \& Saksida, L. M. (2006). Why does brain damage impair memory? a connectionist model of object recognition memory in perirhinal cortex. Journal of Neuroscience, 26, 12186-12197.

Cowell, R. A., Bussey, T. J., \& Saksida, L. M. (2010). Components of recognition memory: Dissociable cognitive processes or just differences in representational complexity? Hippocampus, 20, 1245-1262. 
Cox, G. E., \& Shiffrin, R. M. (in press). A dynamic approach to recognition memory. Psychological Review, .

Criss, A. H., \& Shiffrin, R. M. (2004). Pairs do not suffer interference from other types of pairs or single items in associative recognition. Memory $\mathcal{E}$ Cognition, 32, 1284-1297.

Criss, A. H., \& Shiffrin, R. M. (2005). List discrimination in associative recognition and implications for representation. Journal of Experimental Psychology: Learning, Memory, and Cognition, 31, 1199-1212.

Curran, T., \& Hintzman, D. L. (1995). Violations of the independence assumption in process dissociation. Journal of Experimental Psychology: Learning. Memory, and Cognition, 21, 531-547.

Czerwinski, M., Lightfoot, N., \& Shiffrin, R. M. (1992). Automatization and training in visual search. American Journal of Psychology, 105, 271-315.

Diederich, A., \& Busemeyer, J. R. (2003). Simple matrix methods for analyzing diffusion models of choice probability, choice response time, and simple response time. Journal of Mathematical Psychology, 47, 304-322.

Donkin, C., Little, D. R., \& Houpt, J. W. (2014). Assessing the speed-accuracy tradeoff effect on the capacity of information processing. Journal of Experimental Psychology: Human Perception and Performance, 40, 1183-1202.

Dosher, B. A. (1984). Discriminating preexperimental (semantic) from learned (episodic) associations: A speed-accuracy study. Cognitive Psychology, 16, 519555 .

Dosher, B. A., \& Rosedale, G. (1989). Integrated retrieval cues as a mechanism for priming in retrieval from memory. Journal of Experimental Psychology: General, $118,191-211$.

Dosher, B. A., \& Rosedale, G. (1991). Judgments of semantic and episodic relatedness: Common time-course and failure of segregation. Journal of Memory and Language, $30,125-160$.

Dosher, B. A., \& Rosedale, G. (1997). Configural processing in memory retrieval: Multiple cues and ensemble representations. Cognitive Psychology, 33, 209-265.

Dunn, J. C. (2004). Remember-know: A matter of confidence. Psychological Review, $111,524-542$.

Dunn, J. C. (2008). The dimensionality of the remember-know task: A state-trace analysis. Psychological Review, 115, 426-446.

Eidels, A., Donkin, C., Brown, S. D., \& Heathcote, A. (2010). Converging measures of workload capacity. Psychonomic Bulletin E Review, 17, 763-771. 
Eidels, A., Houpt, J. W., Altieri, N., Pei, L., \& Townsend, J. T. (2011). Nice guys finish fast and bad guys finish last: Facilitatory vs. inhibitory interaction in parallel systems. Journal of Mathematical Psychology, 55, 176-190.

Fific, M., Nosofsky, R. M., \& Townsend, J. T. (2008). Information-processing architectures in multidimensional classification: A validation test of the systems factorial technology. Journal of Experimental Psychology: Human Perception and Performance, 34, 356-375.

Gauthier, I., \& Tarr, M. J. (1997). Becoming a "greeble" expert: Exploring mechanisms for face perception. Vision Research, 37, 1673-1682.

Gillund, G., \& Shiffrin, R. M. (1984). A retrieval model for both recognition and recall. Psychological Review, 91, 1-67.

Goh, J. O. S., Siong, S. C., Park, D., Gutchess, A., Hebrank, A., \& Chee, M. W. L. (2004). Cortical areas involved in object, background, and object-background processing revealed with functional magnetic resonance adaptation. The Journal of Neuroscience, 24, 10223-10228.

Goldstone, R. L. (2000). Unitization during category learning. Journal of Experimental Psychology: Human Perception and Performance, 26, 86-112.

Graf, P., \& Schacter, D. L. (1985). Implicit and explicit memory for new associations in normal and amnesic subjects. Journal of Experimental Psychology: Learning. Memory, and Cognition, 11, 501-518.

Greene, R. L., \& Tussing, A. A. (2001). Similarity and associative recognition. Journal of Memory and Language, 45, 573-584.

Gronlund, S. D., \& Ratcliff, R. (1989). Time course of item and associative information: Implications for global memory models. Journal of Experimental Psychology: Learning, Memory, and Cognition, 15, 846-858.

Haskins, A. L., Yonelinas, A. P., Quamme, J. R., \& Ranganath, C. (2008). Perirhinal cortex supports encoding and familiarity-based recognition of novel associations. Neuron, 59, 554-560.

Hillstrom, A. P., \& Logan, G. D. (1997). Process dissociation, cognitive architecture, and response time: Comments on Lindsay and Jacoby (1994). Journal of Experimental Psychology: Human Perception and Performance, 23, 1561-1578.

Hintzman, D. L. (1988). Judgements of frequency and recognition memory in a multiple-trace memory model. Psychological Review, 95, 528-551.

Hockley, W. E. (1991). Recognition memory for item and associative information: A comparison of forgetting rates. In Relating theory and data: Essays on human memory in honor of Bennet B. Murdock (pp. 227-248).

Hockley, W. E., \& Cristi, C. (1996a). Tests of encoding tradeoffs between item and associative information. Memory $\mathcal{E}$ Cognition, 24, 202-216. 
Hockley, W. E., \& Cristi, C. (1996b). Tests of the separate retrieval of item and associative information using a frequency-judgment task. Memory $\mathcal{E}$ Cognition, 24, 796-811.

Houpt, J. W., Blaha, L. M., McIntire, J. P., Havig, P. R., \& Townsend, J. T. (2014). Systems factorial technology with R. Behavior Research Methods, 46, 307-330.

Houpt, J. W., MacEachern, S. N., Peruggia, M., Townsend, J. T., \& Van Zandt, T. (2016). Semiparametric Bayesian approaches to systems factorial technology. Journal of Mathematical Psychology, 75, 68-85.

Houpt, J. W., \& Townsend, J. T. (2011). An extension of SIC predictions to the Wiener coactive model. Journal of Mathematical Psychology, 55, 267-270.

Humphreys, M. S., Bain, J. D., \& Pike, R. (1989). Different ways to cue a coherent memory system: A theory for episodic, semantic, and procedural tasks. Psychological Review, 96, 208-233.

Jacoby, L. L. (1991). A process dissociation framework: Separating automatic from intentional uses of memory. Journal of Memory and Language, 30, 513-541.

Jones, M., \& Dzhafarov, E. N. (2014). Unfalsifiability and mutual translatability of major modeling schemes for choice reaction time. Psychological Review, 121, 132.

Jou, J. (2010). Can associative information be strategically separated from item information in word-pair recognition? Psychonomic Bulletin $\mathcal{E}$ Review, 17, 778-783.

Kan, I. P., Keane, M. M., Martin, E., Parks-Stamm, E. J., Lewis, L., \& Verfaellie, M. (2011). Implicit memory for novel associations between pictures: Effects of stimulus unitization and aging. Memory $\mathcal{E}$ Cognition, 39, 778-790.

Kelley, R., \& Wixted, J. T. (2001). On the nature of associative information in recognition memory. Journal of Experimental Psychology: Learning, Memory, and Cognition, 27, 701-722.

Kilb, A., \& Naveh-Benjamin, M. (2011). The effects of pure pair repetition on younger and older adults' associative memory. Journal of Experimental Psychology: Learning, Memory, and Cognition, 37, 706-719.

Köhler, W. (1941). On the nature of associations. Proceedings of the American Philosophical Society, 84, 489-502.

Konkle, T., Brady, T. F., Alvarez, G. A., \& Oliva, A. (2010). Conceptual distinctiveness supports detailed visual long-term memory for real-world objects. Journal of Experimental Psychology: General, 139, 558-578.

Kruschke, J. K. (2015). Doing Bayesian Data Analysis: A Tutorial with R, JAGS, and Stan. (2nd ed.). London: Academic Press. 
Kumaran, D., \& Maguire, E. A. (2009). Novelty signals: a window into hippocampal information processing. Trends in Cognitive Sciences, 13, 47-54.

Little, D. R., Eidels, A., Fific, M., \& Wang, T. (2015). Understanding the influence of distractors on workload capacity. Journal of Mathematical Psychology, 68-69, $25-36$.

Little, D. R., Nosofsky, R. M., \& Denton, S. E. (2011). Response-time tests of logicalrule models of categorization. Journal of Experimental Psychology: Learning, Memory, and Cognition, 37, 1-27.

Liu, Y. (1996). Queueing network modeling of elementary mental processes. Psychological Review, 103, 116-136.

Mandler, G. (1980). Recognizing: The judgment of previous occurrence. Psychological Review, 87, 252-271.

McClelland, J. L. (1979). On the time relations of mental processes: An examination of systems of processes in cascade. Psychological Review, 86, 287-330.

McClelland, J. L., \& Chappell, M. (1998). Familiarity breeds differentiation: A subjective-likelihood approach to the effects of experience in recognition memory. Psychological Review, 105, 724-760.

McGee, R. (1980). Imagery and recognition memory: The effects of relational organization. Memory $\mathcal{E}$ Cognition, 8, 394-399.

McKoon, G., \& Ratcliff, R. (2012). Aging and IQ effects on associative recognition and priming in item recognition. Journal of Memory and Language, 66, 416-437.

Mewhort, D. J. K., \& Johns, E. E. (2000). The extralist-feature effect: Evidence against item matching in short-term recognition memory. Journal of Experimental Psychology: General, 129, 262-284.

Miller, J. (1993). A queue-series model for reaction time, with discrete-stage and continuous-flow models as special cases. Psychological Review, 100, 702-715.

Murdock, B. B. (1974). Human Memory: Theory and Data. Hillsdale, NJ: Erlbaum.

Murdock, B. B. (1982). A theory for the storage and retrieval of item and associative information. Psychological Review, 89, 609-626.

Murnane, K., \& Shiffrin, R. M. (1991). Interference and the representation of events in memory. Journal of Experimental Psychology: Learning, Memory, and Cognition, $17,855-874$.

Naveh-Benjamin, M. (2000). Adult age differences in memory performance: Tests of an associative deficit hypothesis. Journal of Experimental Psychology: Learning, Memory, and Cognition, 26, 1170-1187. 
Naveh-Benjamin, M., Hussain, Z., Guez, J., \& Bar-On, M. (2003). Adult age differences in episodic memory: Further support for an associative-deficit hypothesis. Journal of Experimental Psychology: Learning, Memory, and Cognition, 29, 826837.

Naveh-Benjamin, M., Shing, Y. L., Kilb, A., Werkle-Bergner, M., Lindenberger, U., $\&$ Li, S. (2009). Adult age differences in memory for name-face associations: The effects of intentional and incidental learning. Memory, 17, 220-232.

Nelson, A. B., \& Shiffrin, R. M. (2013). The co-evolution of knowledge and event memory. Psychological Review, 120, 356-394.

Nobel, P. A., \& Shiffrin, R. M. (2001). Retrieval processes in recognition and cued recall. Journal of Experimental Psychology: Learning, Memory, and Cognition, 27, 384-413.

Old, S. R., \& Naveh-Benjamin, M. (2008). Memory for people and their actions: Further evidence for an age-related associative deficit. Psychology and Aging, 23, 467-472.

Paivio, A. (1969). Mental imagery in associative learning and memory. Psychological Review, 76, 241-263.

Parks, C. M., \& Yonelinas, A. P. (2015). The importance of unitization for familiaritybased learning. Journal of Experimental Psychology: Learning, Memory, and Cognition, 41, 881-903.

Peirce, J. W. (2007). PsychoPy-psychophysics software in Python. Journal of Neuroscience Methods, 162, 8-13.

Plummer, M. (2013). JAGS: Just another Gibbs sampler. URL: http://mcmc-jags . sourceforge.net/.

Pratte, M. S., \& Rouder, J. N. (2012). Assessing the dissociability of recollection and familiarity in recognition memory. Journal of Experimental Psychology: Learning, Memory, and Cognition, 38, 1591-1607.

Prior, A., \& Bentin, S. (2003). Incidental formation of episodic associations: The importance of sentential context. Memory $\mathcal{E}$ Cognition, 31, 306-316.

Prior, A., \& Bentin, S. (2008). Word associations are formed incidentally during sentential semantic integration. Acta Psychologica, 127, 57-71.

Ratcliff, R., \& McKoon, G. (1988). A retrieval theory of priming in memory. Psychological Review, 95, 385-408.

Ratcliff, R., \& Murdock, B. B. (1976). Retrieval processes in recognition memory. Psychological Review, 83, 190-214.

Ratcliff, R., Thapar, A., \& McKoon, G. (2011). Effects of aging and IQ on item and associative memory. Journal of Experimental Psychology: General, 140, 464-487. 
Ratcliff, R., Van Zandt, T., \& McKoon, G. (1995). Process dissociation, single-process theories, and recognition memory. Journal of Experimental Psychology: General, $124,352-374$.

Rhodes, M. G., Castel, A. D., \& Jacoby, L. L. (2008). Associative recognition of face pairs by younger and older adults: The role of familiarity-based processing. Psychology and Aging, 23, 239-249.

Rotello, C. M., \& Heit, E. (2000). Associative recognition: A case of recall-to-reject processing. Memory $\mathcal{E}$ Cognition, 28, 907-922.

Rouder, J. N., Lu, J., Speckman, P. L., Sun, D., \& Jiang, Y. (2005). A hierarchical model for estimating response time distributions. Psychonomic Bulletin $\mathcal{F}$ Review, $12,195-223$.

Rugg, M. D., \& Curran, T. (2007). Event-related potentials and recognition memory. Trends in Cognitive Sciences, 11, 251-257.

Schweickert, R. (1989). Separable effects of factors on activation functions in discrete and continuous models: $d^{\prime}$ and evoked potentials. Psychological Bulletin, 106, $318-$ 328.

Shiffrin, R. M., \& Lightfoot, N. (1997). Perceptual learning of alphanumeric-like characters. In R. L. Goldstone, P. G. Schyns, \& D. L. Medin (Eds.), The psychology of learning and motivation (pp. 83-126). San Diego, CA: Academic Press volume 36.

Shiffrin, R. M., \& Steyvers, M. (1997). A model for recognition memory: REMretrieving effectively from memory. Psychonomic Bulletin $\mathcal{E}$ Review, 4, 145-166.

Smith, E. E., \& Haviland, S. E. (1972). Why words are perceived more accurately than nonwords: Inference versus unitization. Journal of Experimental Psychology, 92, 59-64.

Smith, L. B., Suanda, S. H., \& Yu, C. (2014). The unrealized promise of infant statistical word-referent learning. Trends in Cognitive Sciences, 18, 251-258.

Smith, L. B., \& Yu, C. (2008). Infants rapidly learn word-referent mappings via crosssituational statistics. Cognition, 106, 1558-1568.

Speer, N. K., \& Curran, T. (2007). ERP correlates of familiarity and recollection processes in visual associative recognition. Brain Research, 1174, 97-109.

Staresina, B. P., \& Davachi, L. (2010). Object unitization and associative memory formation are supported by distinct brain regions. Journal of Neuroscience, 30, 9890-9897.

Stewart, N., \& Brown, G. D. (2005). Similarity and dissimilarity as evidence in perceptual categorization. Journal of Mathematical Psychology, 49, 403-409.

Thomson, D. M., \& Tulving, E. (1970). Associative encoding and retrieval: Weak and strong cues. Journal of Experimental Psychology, 86, 255-262. 
Townsend, J. T., \& Altieri, N. (2012). An accuracy-response time capacity assessment function that measures performance against standard parallel predictions. Psychologial Review, 119, 500-516.

Townsend, J. T., \& Fikes, T. (1995). A beginning quantitative taxonomy of cognitive activation systems and application to continuous flow processes. Technical Report 131 Indiana University Bloomington, Cognitive Science Program.

Townsend, J. T., \& Nozawa, G. (1995). Spatio-temporal properties of elementary perception: An investigation of parallel, serial, and coactive theories. Journal of Mathematical Psychology, 39, 321-359.

Townsend, J. T., \& Thomas, R. D. (1994). Stochastic dependencies in parallel and serial models: Effects on systems factorial interactions. Journal of Mathematical Psychology, 38, 1-34.

Townsend, J. T., \& Wenger, M. J. (2004). A theory of interactive parallel processing: New capacity measures and predictions for a response time inequality series. Psychologial Review, 111, 1003-1035.

Townsend, J. T., \& Wenger, M. J. (2015). On the dynamic perceptual characteristics of Gestalten: Theory-based methods. In J. Wagemans (Ed.), The Oxford Handbook of Perceptual Organization. Oxford: Oxford University Press.

Tulving, E., \& Thompson, D. M. (1973). Encoding specificity and retrieval processes in episodic memory. Psychological Review, 80, 352-373.

Tversky, A. (1977). Features of similarity. Psychological Review, 84, 327-352.

Wagemans, J., Elder, J. H., Kubovy, M., Palmer, S. E., Peterson, M. A., Singh, M., \& von der Heydt, R. (2012a). A century of Gestalt psychology in visual perception: I. perceptual grouping and figure-ground organization. Psychological Bulletin, 138, $1172-1217$.

Wagemans, J., Feldman, J., Gepshtein, S., Kimchi, R., Pomerantz, J. R., van der Helm, P. A., \& van Leeuwen, C. (2012b). A century of Gestalt psychology in visual perception: II. conceptual and theoretical foundations. Psychological Bulletin, 138, $1218-1252$.

Wickelgren, W. A., \& Corbett, A. T. (1977). Associative interference and retrieval dynamics in yes-no recall and recognition. Journal of Experimental Psychology: Human Learning and Memory, 3, 189-202.

Wixted, J. T. (2007). Dual-process theory and signal-detection theory of recognition memory. Psychological Review, 114, 152-176.

Yang, H., Fific, M., \& Townsend, J. T. (2014). Survivor interaction contrast wiggle predictions of parallel and serial models for an arbitrary number of processes. Journal of Mathematical Psychology, 58, 21-32. 
Table A.1: Sets of parameter values used to simulate systems of accumulators. Values were chosen to span the range of possible behavior for each architecture. For each processing architecture, we obtained predictions for all combinations of parameter values subject to the constraints that $u_{H}^{1}>u_{L}^{1}$ and $u_{H}^{2}>u_{L}^{2}$, reflecting the assumption that strength manipulations are effective. The $k$ parameter only applies to serial architectures in which the order of processing may vary.

\begin{tabular}{rl}
\hline Parameter & Values \\
\hline$u_{H}^{1}$ & $0.06,0.07,0.08$ \\
$u_{L}^{1}$ & $0.05,0.06,0.07$ \\
$u_{H}^{2}$ & $0.06,0.07,0.08$ \\
$u_{L}^{2}$ & $0.05,0.06,0.07$ \\
$B_{C}^{1}$ & $2,3,4$ \\
$B_{E}^{1}$ & $2,3,4$ \\
$B_{C}^{2}$ & $2,3,4$ \\
$B_{E}^{2}$ & $2,3,4$ \\
$k$ & $0.5,0.75,1$ \\
\hline
\end{tabular}

Yonelinas, A. P. (1997). Recognition memory ROCs for item and associative information: The contribution of recollection and familiarity. Memory $\mathcal{E}$ Cognition, 25, $747-763$.

Zacks, J. M., \& Tversky, B. (2001). Event structure in perception and cognition. Psychological Bulletin, 127, 3-21.

\section{Appendix A. Properties of interacting processes}

Because we could not guarantee that our repetition manipulations would selectively influence item and associative strength, we augmented analytical predictions for the SIC (Townsend \& Nozawa, 1995) with simulations that examine the consequences of pooling between the two processes (for additional simulation results in high-accuracy situations, see Eidels et al. 2011).

\section{A.1. Simulations}

In our simulations, we assume that a single retrieval process is described by a pair of parallel accumulators, one leading toward a correct response and one leading toward an error. On each unit of time, the correct accumulator is incremented with probability $u$ and the error accumulator is incremented with probability $v$. A correct response results when the correct accumulator accrues $B_{C}$ total counts while an error response results if the error accumulator accrues a total of $B_{E}$ counts; the first accumulator to reach its criterion determines the response and response time. Accumulators begin with zero counts and can only accrue counts; counts are never lost. We additionally adopt the simplifying assumption that the probability that the error accumulator is incremented is inversely related to the probability with which the correct accumulator is incremented such that $v=0.09-u$. 
Two independent processes are modeled as two pairs of parallel accumulators of the kind just described. Each process has its own set of correct and error criteria, $B_{C}^{1}$ and $B_{E}^{1}$ for the first process and $B_{C}^{2}$ and $B_{E}^{2}$ for the second. In addition, each process has its own rate of correct accumulation when it is high strength $(\mathrm{H})$ and low strength (L), $u_{H}^{1}$ and $u_{L}^{1}$ for the first process and $u_{H}^{2}$ and $u_{L}^{2}$ for the second. For each processing architecture, we obtained predictions from a wide range of parameter values, as given in Table A.1, in order to establish the range of behavior each architecture could generate.

Analytic simulation results were obtained via matrix methods (Diederich \& Busemeyer 2003). While the precise implementation depended on the simulated architecture, as described below, the general idea is to define the state space $\mathbf{X}$ of the accumulators and use the increment probabilities $u^{1}$ and $u^{2}$ and stopping rule (self-terminating or exhaustive) to determine the probability of transitioning from one part of the state space $x_{i} \in \mathbf{X}$ to another $x_{j} \in \mathbf{X}$, expressed via a transition matrix $Q$. There are two absorbing states in which the system has finished processing and the transition probabilities are zero: One set of states $R_{C} \subset \mathbf{X}$ corresponds to a correct response, where the appropriate accumulators have reached criterion; another set $R_{E} \subset \mathbf{X}$ corresponds to error responses, where a different set of accumulators have reached criterion. Starting with an initial state distribution $Z_{0}$ concentrated entirely over the state where all accumulators have zero counts, we iteratively apply the transition matrix $Z_{n}=Q Z_{n-1}$ from one time-step to the next and track the total amount of probability mass residing in $R_{C}$ and $R_{E}$ at each time-step, yielding the finishing time distributions $F_{C}[n]$ and $F_{E}[n]$.

\section{A.1.1. Independent parallel processing}

In the independent parallel model, there are a total of four accumulators corresponding to correct and error information for each process. Thus, the state space $\mathbf{X}$ is defined by four dimensions, $\left[0, B_{C}^{1}\right] \times\left[0, B_{E}^{1}\right] \times\left[0, B_{C}^{2}\right] \times\left[0, B_{E}^{2}\right]$, where any given state is defined by the number of counts in each accumulator, $x_{i}=\left\{v_{C}^{1}, v_{E}^{1}, v_{C}^{2}, v_{E}^{2}\right\}$. For self-terminating processing, the absorbing states corresponding to correct responses are those in which $v_{C}^{1}=B_{C}^{1}$ or $v_{C}^{2}=B_{C}^{2}$ while either $v_{E}^{1}<B_{E}^{1}$ or $v_{E}^{2}<B_{E}^{2}$. For exhaustive processing, the absorbing states corresponding to correct responses are those in which both $v_{C}^{1}=B_{C}^{1}$ and $v_{C}^{2}=B_{C}^{2}$ and both $v_{E}^{1}<B_{E}^{1}$ and $v_{E}^{2}<B_{E}^{2}$.

\section{A.1.2. Independent serial processing}

Unlike in parallel processing, in serial processing only one pair of accumulators is active at any one time. It is possible that one process is chosen to be the first process more often; we denote the probability that process 1 goes before process 2 with parameter $k$. Interactions occur if the first process provides information to the second process. In such a case, one or both accumulators continue to accept input from the first process and combine it with the second. Hence our claim in the main text that interactions entail parallel processing - for at least one accumulator, both processes continue to operate concurrently.

To obtain predictions for serial processes, we followed a similar approach to parallel processing. We used the same matrix methods to obtain predictions for the finishing times for each process (i.e., each pair of correct and error accumulators) separately, 
conditioned on the order of processing. $F_{C}^{11}[n]$ and $F_{E}^{11}[n]$ are the finishing time distributions for process 1 when it is first, $F_{C}^{12}[n]$ and $F_{E}^{12}[n]$ are the finishing time distributions for process 1 when it is second, $F_{C}^{21}[n]$ and $F_{E}^{21}[n]$ are the finishing time distributions for process 2 when it is first, and $F_{C}^{22}[n]$ and $F_{E}^{22}[n]$ are the finishing time distributions for process 2 when it is second. Letting lower case $f[n]$ 's denote the finishing time mass at step $n$ (i.e., the difference $F[n+1]-F[n]$ ), we can then express the finishing time distributions under self-terminating (ST) or exhaustive (EX) processing:

$$
\begin{aligned}
& F_{S T}[n]=\sum_{i=0}^{n}\left\{k\left(f_{C}^{11}[i]+\sum_{j=0}^{i} f_{E}^{11}[j] f_{C}^{22}[i-j]\right)+(1-k)\left(f_{C}^{21}[i]+\sum_{j=0}^{i} f_{E}^{21}[j] f_{C}^{12}[i-j]\right)\right\} \\
& F_{E X}[n]=\sum_{i=0}^{n}\left\{k \sum_{j=0}^{i} f_{C}^{11}[j] f_{C}^{22}[i-j]+(1-k) \sum_{j=0}^{i} f_{C}^{21}[j] f_{C}^{12}[i-j]\right\} .
\end{aligned}
$$

In all cases, the state spaces for the component processes $\mathbf{X}^{\mathbf{1}}$ and $\mathbf{X}^{\mathbf{2}}$ are independent of processing order and are defined over the respective correct and error accumulators, i.e., $\left[0, B_{C}^{1}\right] \times\left[0, B_{E}^{1}\right]$ and $\left[0, B_{C}^{2}\right] \times\left[0, B_{E}^{2}\right]$.

Under independent serial processing, the order of processing does not affect either process. When process 1 is operating, its correct accumulator accrues a count with probability $u^{1}$ and its error accumulator accrues a count with probability $v^{1}$, similarly for process 2 .

\section{A.1.3. Facilitatory processing}

As mentioned in the main text, when processes are allowed to pool their resources, they must be considered parallel processes, since interactions can only occur if the two processes overlap in time. Nonetheless, it is still possible that one process may begin earlier than the other.

If the processes begin at the same time, there are three accumulators, one corresponding to correct information and two corresponding to error information. The response threshold for the correct accumulator is $B_{C}^{1}+B_{C}^{2}$ and the probability of accumulating a count on the correct accumulator is $1-\left(1-u^{1}\right)\left(1-u^{2}\right)$, i.e., the probability that either process generates a correct count. The state space $\mathbf{X}$ is defined by three dimensions, $\left[0, B_{C}^{1}+B_{C}^{2}\right] \times\left[0, B_{E}^{1}\right] \times \times\left[0, B_{E}^{2}\right]$, where any given state is defined by the number of counts in each accumulator, $x_{i}=\left\{v_{C}, v_{E}^{1}, v_{E}^{2}\right\}$. For self-terminating processing, the absorbing states corresponding to correct responses are those in which $v_{C}=B_{C}^{1}+B_{C}^{2}$ while either $v_{E}^{1}<B_{E}^{1}$ or $v_{E}^{2}<B_{E}^{2}$. For exhaustive processing, the absorbing states corresponding to correct responses are those in which $v_{C}=B_{C}^{1}+B_{C}^{2}$ and both $v_{E}^{1}<B_{E}^{1}$ and $v_{E}^{2}<B_{E}^{2}$.

To model interactive systems where one process begins before the other ("continuousflow" systems), we treat the first process as if it were the first stage of independent serial processing (thus starting order is governed by probability $k$, as in independent processing). The first process is thus allowed to terminate whenever its correct or incorrect accumulator reaches its respective criterion. If the second process is then engaged (i.e., under an exhaustive stopping rule), the probability of accumulating a correct count for 
that process is augmented by the first: although the error accumulator for the second process accumulates counts as usual, the correct accumulator accrues counts with probability $1-\left(1-u^{1}\right)\left(1-u^{2}\right)$. Thus, the first process "spills over" into the second, acting to facilitate the second process even though they start at different times.

\section{A.1.4. Inhibitory processing}

As with facilitatory processing, we consider two situations: one in which both processes begin at the same time and another in which they can start at different times.

If the processes begin simultaneously, there are three accumulators, one corresponding to error information and two corresponding to correct information. The response threshold for the error accumulator is $B_{E}^{1}+B_{E}^{2}$ and the probability of accumulating a count on the error accumulator is $1-\left(1-v^{1}\right)\left(1-v^{2}\right)$, i.e., the probability that either process generates an erroneous count. The state space $\mathbf{X}$ is defined by three dimensions, $\left[0, B_{C}^{1}\right] \times\left[0, B_{C}^{2}\right] \times\left[0, B_{E}^{1}+B_{E}^{2}\right]$, where any given state is defined by the number of counts in each accumulator, $x_{i}=\left\{v_{C}^{1}, v_{C}^{2}, v_{E}\right\}$. For self-terminating processing, the absorbing states corresponding to correct responses are those in which either $v_{C}^{1}=B_{C}^{1}$ or $v_{C}^{2}=B_{C}^{2}$ while $v_{E}<B_{E}$. For exhaustive processing, the absorbing states corresponding to correct responses are those in which both $v_{C}^{1}=B_{C}^{1}$ and $v_{C}^{2}=B_{C}^{2}$ while $v_{E}<B_{E}^{1}+B_{E}^{2}$.

As in facilitatory processing, if the two processes begin at different times, whichever process is first proceeds as if it were the first stage of independent serial processing. Whichever process goes second accumulates counts on the error accumulator with probability $1-\left(1-v^{1}\right)\left(1-v^{2}\right)$.

\section{A.1.5. Coactive processing}

When the two processes begin at the same time, there are two accumulators, one corresponding to correct information and one to error information. The response threshold for the correct accumulator is $B_{C}^{1}+B_{C}^{2}$ and for the error accumulator is $B_{E}^{1}+B_{E}^{2}$. The probability of accumulating a count on the correct accumulator is $1-\left(1-u^{1}\right)\left(1-u^{2}\right)$ while the probability of accumulating a count on the error accumulator is $1-\left(1-v^{1}\right)(1-$ $\left.v^{2}\right)$. The state space $\mathbf{X}$ is defined by two dimensions, $\left[0, B_{C}^{1}+B_{C}^{2}\right] \times\left[0, B_{E}^{1}+B_{E}^{2}\right]$, where any given state is defined by the number of counts in each accumulator, $x_{i}=\left\{v_{C}, v_{E}\right\}$. There is only one stopping rule possible in coactive processing, so the absorbing states corresponding to correct responses are those in which $v_{C}=B_{C}^{1}+B_{C}^{2}$ and $v_{E}<B_{E}^{1}+B_{E}^{2}$.

When the two processes begin at different times, the first process operates as if it were the first stage of independent serial processing, but whichever process goes second accumulates correct counts with probability $1-\left(1-u^{1}\right)\left(1-u^{2}\right)$ and error counts with probability $1-\left(1-v^{1}\right)\left(1-v^{2}\right)$. Coactive processing implies that there is only one possible stopping rule, which in the case of different starting times corresponds to exhaustive processing since both processes must be engaged before a decision can be made.

\section{A.1.6. Determining the range of possible forms}

For visualization purposes (Figure 3), we determined the range of possible functional forms that each of these processing types could produce in terms of the largest 
positive and negative deflections predicted for each architecture, i.e., $|\max f(x)|-$ $|\min f(x)|$. The upper limit for a particular processing type is the function produced by the parameters that maximizes this quantity while the lower limit is the predicted function that minimizes this quantity.

\section{A.2. Analytic results}

To better understand the situations in which processing architectures can produce SIC's that differ from those obtained under the assumption of selective influence, we present a set of inequalities that describe how interactions between two processes can affect SIC predictions. The following extends to RT distributions the results of Townsend \& Thomas (1994) on the properties of mean response time in the absence of selective influence.

\section{A.2.1. Inhibitory parallel self-terminating processing}

Assuming self-terminating processing, the SIC is given by

$$
\begin{aligned}
S I C_{P S T}(t)= & {\left[S_{L L}(t)-S_{L H}(t)\right]-\left[S_{H L}(t)-S_{H H}(t)\right] } \\
= & {\left[F_{L H}(t)-F_{L L}(t)\right]-\left[F_{H H}(t)-F_{H L}(t)\right] } \\
= & {\left[F_{L}(t)+G_{H \mid L}(t)-F_{L}(t) G_{H \mid L}(t)-F_{L}(t)-G_{L \mid L}(t)+F_{L}(t) G_{L \mid L}(t)\right] } \\
& \quad-\left[F_{H}(t)+G_{H \mid H}(t)-F_{H}(t) G_{H \mid H}(t)-F_{H}(t)-G_{L \mid H}(t)+F_{H}(t) G_{L \mid H}(t)\right] \\
= & {\left[1-F_{L}(t)\right]\left[G_{H \mid L}(t)-G_{L \mid L}(t)\right]-\left[1-F_{H}(t)\right]\left[G_{H \mid H}(t)-G_{L \mid H}(t)\right] }
\end{aligned}
$$

where $F_{L}(t)$ and $F_{H}(t)$ are the finishing time distribution functions for the first process at low $(\mathrm{L})$ and high $(\mathrm{H})$ levels of strength, $G_{H \mid L}(t)$ and $G_{L \mid L}(t)$ are the finishing time distributions for the second process at low and high levels of strength given that the first process is low strength, similarly for $G_{H \mid H}(t)$ and $G_{L \mid H}(t)$.

If the two processes are independent, $G_{H}(t)=G_{H \mid L}(t)=G_{H \mid H}(t)$ and $G_{L}(t)=$ $G_{L \mid L}(t)=G_{L \mid H}(t)$, giving

$$
\begin{aligned}
S I C_{P S T}(t) & =\left[1-F_{L}(t)\right]\left[G_{H}(t)-G_{L}(t)\right]-\left[1-F_{H}(t)\right]\left[G_{H}(t)-G_{L}(t)\right] \\
& =\left[G_{H}(t)-G_{L}(t)\right]\left[F_{H}(t)-F_{L}(t)\right] \\
& \geq 0
\end{aligned}
$$

because, by assumption, $F_{H}(t) \geq F_{L}(t)$ and $G_{H}(t) \geq G_{L}(t)$.

However, if the two processes are not independent, then it is possible for the SIC to lie above (or below) zero, depending on the nature of the interaction:

$$
\begin{aligned}
0 & \geq\left[1-F_{L}(t)\right]\left[G_{H \mid L}(t)-G_{L \mid L}(t)\right]-\left[1-F_{H}(t)\right]\left[G_{H \mid H}(t)-G_{L \mid H}(t)\right] \\
\frac{G_{H \mid H}(t)-G_{L \mid H}(t)}{G_{H \mid L}(t)-G_{L \mid L}(t)} & \geq \frac{1-F_{L}(t)}{1-F_{H}(t)} \geq 1
\end{aligned}
$$

This inequality states that a parallel self-terminating process can produce a negative SIC if the speedup of the second process when the first process is high strength is sufficiently greater than the speedup of the second process when the first process is low strength. Put another way, a substantial speedup can occur only when both processes 
are high strength - if even one is low strength, processing is slow. Such a situation occurs when low strength on one process inhibits high strength in the other process or, equivalently, processing capacity is severely limited such that a speedup occurs only when both processes are high strength.

\section{A.2.2. Facilitatory parallel exhaustive processing}

Assuming exhaustive processing, the SIC is given by

$$
\begin{aligned}
\operatorname{SIC}_{P E X}(t) & =\left[S_{L L}(t)-S_{L H}(t)\right]-\left[S_{H L}(t)-S_{H H}(t)\right] \\
& =\left[F_{L H}(t)-F_{L L}(t)\right]-\left[F_{H H}(t)-F_{H L}(t)\right] \\
& =\left[F_{L}(t) G_{H \mid L}(t)-F_{L}(t) G_{L \mid L}(t)\right]-\left[F_{H}(t) G_{H \mid H}(t)-F_{H}(t) G_{L \mid H}(t)\right] \\
& =F_{L}(t)\left[G_{H \mid L}(t)-G_{L \mid L}(t)\right]-F_{H}(t)\left[G_{H \mid H}(t)-G_{L \mid H}(t)\right]
\end{aligned}
$$

where $F_{L}(t)$ and $F_{H}(t)$ are the finishing time distribution functions for the first process at low (L) and high (H) levels of strength, $G_{H \mid L}(t)$ and $G_{L \mid L}(t)$ are the finishing time distributions for the second process at low and high levels of strength given that the first process is low strength, similarly for $G_{H \mid H}(t)$ and $G_{L \mid H}(t)$.

If the two processes are independent, $G_{H}(t)=G_{H \mid L}(t)=G_{H \mid H}(t)$ and $G_{L}(t)=$ $G_{L \mid L}(t)=G_{L \mid H}(t)$, giving

$$
\begin{aligned}
S I C_{P E X}(t) & =F_{L}(t)\left[G_{H}(t)-G_{L}(t)\right]-F_{H}(t)\left[G_{H}(t)-G_{L}(t)\right] \\
& =\left[F_{L}(t)-F_{H}(t)\right]\left[G_{H}(t)-G_{L}(t)\right] \\
& \leq 0
\end{aligned}
$$

because, by assumption, $F_{L}(t) \leq F_{H}(t)$ and $G_{H}(t) \geq G_{L}(t)$.

However, if the two processes are not independent, then it is possible for the SIC to lie above (or below) zero, depending on the nature of the interaction:

$$
\begin{aligned}
0 & \leq F_{L}(t)\left[G_{H \mid L}(t)-G_{L \mid L}(t)\right]-F_{H}(t)\left[G_{H \mid H}(t)-G_{L \mid H}(t)\right] \\
F_{H}(t)\left[G_{H \mid H}(t)-G_{L \mid H}(t)\right] & \leq F_{L}(t)\left[G_{H \mid L}(t)-G_{L \mid L}(t)\right] \\
1 \leq \frac{F_{H}(t)}{F_{L}(t)} & \leq \frac{\left[G_{H \mid L}(t)-G_{L \mid L}(t)\right]}{\left[G_{H \mid H}(t)-G_{L \mid H}(t)\right]}
\end{aligned}
$$

The right hand ratio reflects the relative speedup of the second process as it moves from low to high strength when the first process is low versus high strength. Thus, the inequality states that a parallel exhaustive process can produce a positive SIC if this speedup is much greater when the first process is low strength than when it is high strength. Such a situation occurs if the two processes facilitate one another such that information from the first process "leaks" into the second process. In this case, high strength from the first process leaks into the second, making variations in the strength of the second process less effective. This comports exactly with our simulation results in which exhaustive processing allows for positive SIC's only in the presence of facilitatory interactions. 
$\mathrm{I}^{+} \mathrm{A}^{+}$
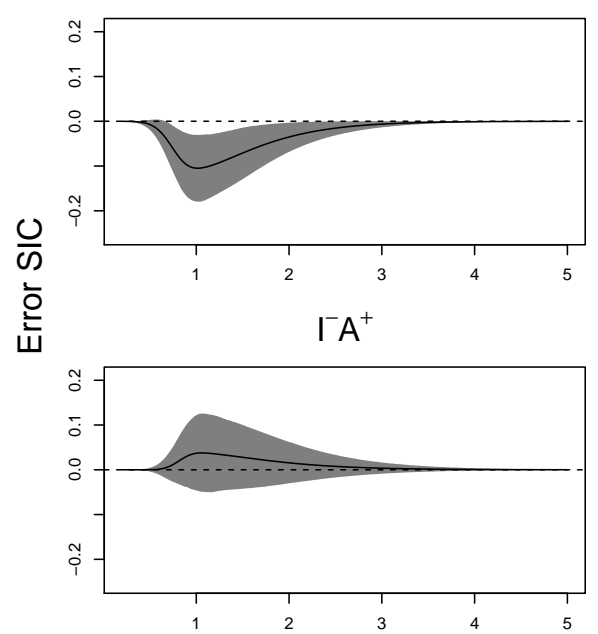

$I^{+} A^{-}$
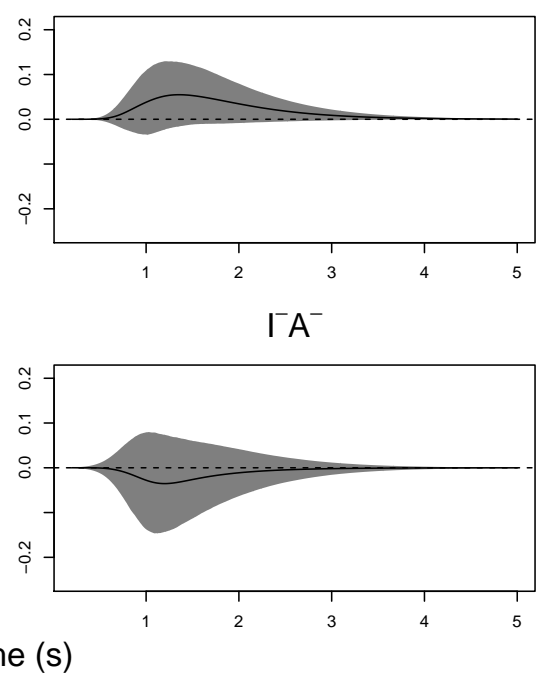

Figure B.1: SIC functions for error responses. Solid lines are posterior means while shaded regions are $95 \%$ credible intervals.

\section{Appendix B. SIC's for error responses}

Although the theory behind the SIC focuses on correct responses, as we did in the main text, it is possible to compute SIC's for error response times as well (we label these "ESIC" functions). Although error responses can arise for many reasons, it is reasonable to assume that they are "true errors" in that participants generate errors when memory evidence is poor, rather than by simple lapses in attention. To the extent that errors arise from the same processes that generate correct responses, the ESIC's should "mirror" the SIC's obtained for correct responses. That is, the ESIC's for false alarms (giving a positive response to $I^{+} A^{-}, I^{-} A^{+}$, or $I^{-} A^{-}$pairs) should resemble those for hits (correct positive responses to $I^{+} A^{+}$pairs) and the ESIC's for misses (giving a negative response to $I^{+} A^{+}$pairs) should resemble those for correct rejections (correct negative responses to $I^{+} A^{-}, I^{-} A^{+}$, and $I^{-} A^{-}$pairs).

The ESIC functions are defined as

$$
\begin{aligned}
& E S I C_{I^{+} A^{+}}(t)=\left[E S_{I_{L}^{+} A_{L}^{+}}(t)-E S_{I_{L}^{+} A_{H}^{+}}(t)\right]-\left[E S_{I_{H}^{+} A_{L}^{+}}(t)-E S_{I_{H}^{+} A_{H}^{+}}(t)\right] \\
& E S I C_{I^{+} A^{-}}(t)=\left[E S_{I_{H}^{+} A_{H}^{-}}(t)-E S_{I_{H}^{+} A_{L}^{-}}(t)\right]-\left[E S_{I_{L}^{+} A_{H}^{-}}(t)-E S_{I_{L}^{+} A_{L}^{-}}(t)\right] \\
& E S I C_{I^{-} A^{+}}(t)=\left[E S_{I_{H}^{-} A_{H}^{+}}(t)-E S_{I_{H}^{-} A_{L}^{+}}(t)\right]-\left[E S_{I_{L}^{-} A_{H}^{+}}(t)-E S_{I_{L}^{-} A_{L}^{+}}(t)\right] \\
& E S I C_{I^{-} A^{-}}(t)=\left[E S_{I_{H}^{-} A_{H}^{-}}(t)-E S_{I_{H}^{-} A_{L}^{-}}(t)\right]-\left[E S_{I_{L}^{-} A_{H}^{-}}(t)-E S_{I_{L}^{-} A_{L}^{-}}(t)\right]
\end{aligned}
$$

where each $E S . .(t)$ is the survivor function of the error RT's in that condition. The posterior distributions over these functions are shown in Figure B.1 The error SIC's are, indeed, mirrored relative to the correct SIC's in each condition as one would expect 


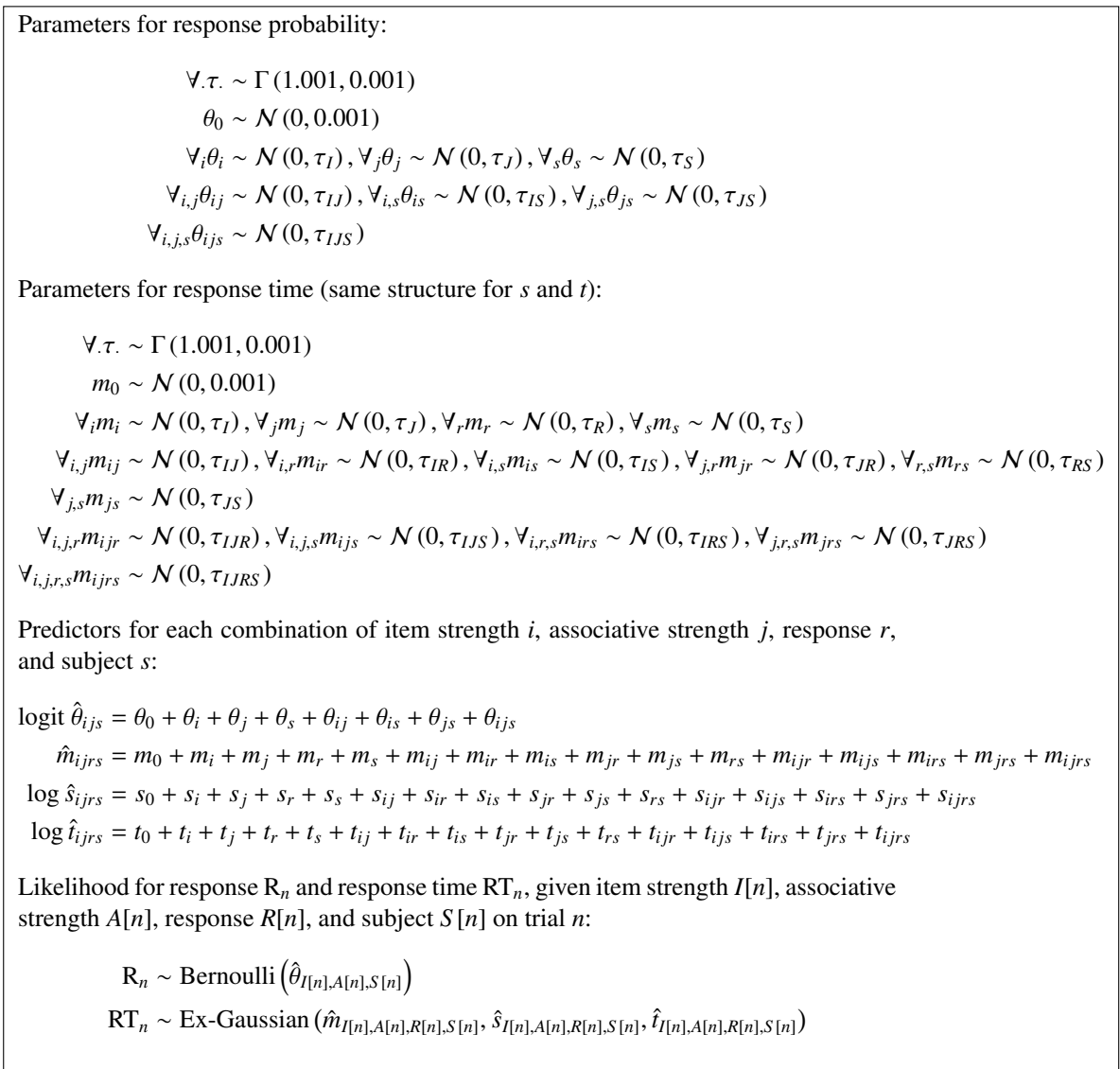

Figure C.1: Hierarchical model structure for estimating response probabilities and RT distributions.

if the same processes lead toward the same responses (regardless of correctness).

\section{Appendix C. Hierarchical RT model}

The hierarchical model structure used to estimate individual and group-level response probabilities and RT distributions is depicted in Figure C.1 To ensure that our model of response probabilities and RT distributions accurately represents the data, we report the posterior predictive distributions for mean response probabilities and RT's in Figure C.2

\section{Appendix D. Linear ballistic accumulator modeling}

As described in the main text, we simultaneously estimated LBA parameters and retrieval architecture according to the hierarchical Bayesian model depicted in Figure D.1 The retrieval architecture for each participant, $M_{s}$, is sampled from one of 

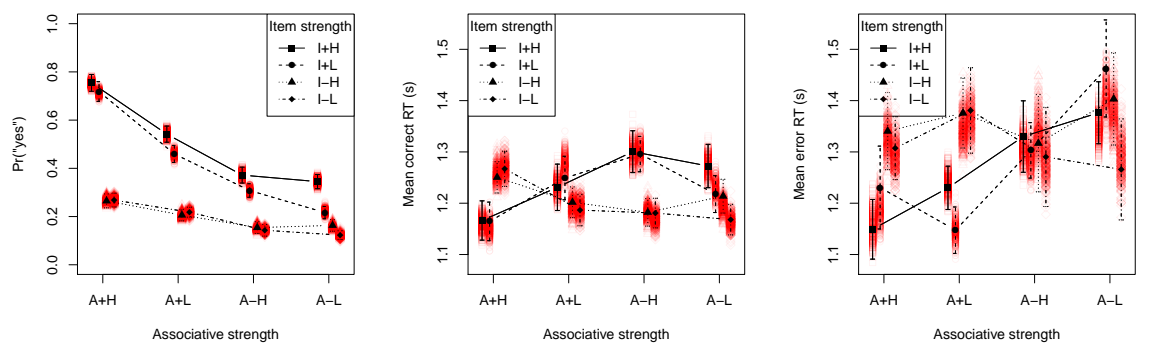

Figure C.2: Posterior predictive distributions from our hierarchical RT model. Observed data are in black, showing means and $95 \%$ confidence intervals about the mean. Red points are posterior samples of the mean.

Table D.1: Posterior modes and 95\% credible intervals for the mean of various LBA parameters. Note that these values are on the "natural" scale of each parameter, rather than on log- or logistic-transformed scales.

\begin{tabular}{rlllll}
\hline & $\begin{array}{l}\text { Boundary } \\
\text { separation }\left(B_{s}\right)\end{array}$ & Bias $\left(w_{s}\right)$ & $\begin{array}{l}\text { Startpoint } \\
\text { variability }\left(A_{s}\right)\end{array}$ & $\begin{array}{l}\text { Residual } \\
\text { time }\left(R_{s}\right)\end{array}$ & $\begin{array}{l}\text { Contaminant } \\
\text { probability }\left(c_{s}\right)\end{array}$ \\
\hline Mode & 2.72 & 0.516 & 2.85 & 0.202 & 0.024 \\
$2.5 \%$ quantile & 2.52 & 0.506 & 2.59 & 0.162 & 0.019 \\
$97.5 \%$ quantile & 3.06 & 0.526 & 3.12 & 0.232 & 0.029 \\
\hline
\end{tabular}

seven possibilities, which we label in terms of the processes leading toward a positive ("yes") response: independent parallel self-terminating (PST), independent parallel exhaustive (PEX), facilitatory exhaustive (FEX), facilitatory self-terminating (FST), inhibitory exhaustive (IEX), inhibitory self-terminating (IST), or coactive (COA). The likelihood $L_{M_{s}}$ of each participant's responses and response times is therefore computed differently depending on which of these architectures was sampled, as described in the main text.

\section{D.1. Posterior predictive}

To verify that this LBA model accurately represents the data, we depict posterior predictive samples in Figure D.2.

\section{D.2. Distributions over other parameters}

The parameters that were of theoretical interest were the drift rates, representing the memory evidence entering into the accumulators, and the retrieval architecture, reflecting how those inputs were combined and used to reach a decision. However, for completeness, we also report the distributions of the posterior means for the other LBA parameters, namely, boundary separation $\left(B_{s}\right)$, startpoint variability $\left(A_{s}\right)$, bias $\left(w_{s}\right)$, contaminant probability $\left(c_{s}\right)$, and residual time $\left(R_{s}\right)$ in Table D.1.

\section{D.3. Individual correlations}

Given that there are interactions between item and associative information in terms of both drift rates (i.e., evidence derived from encoding) and retrieval processes, do 
Retrieval architecture: $\pi \sim$ Dirichlet (1)

Boundary separation: $\log B_{0} \sim \mathcal{N}(0,0.001), \tau_{B} \sim \Gamma(1.001,0.001)$

Startpoint variability: $\log A_{0} \sim \mathcal{N}(0,0.001), \tau_{A} \sim \Gamma(1.001,0.001)$

Bias: $\operatorname{logit} w_{0} \sim \mathcal{N}(0,0.001), \tau_{w} \sim \Gamma(1.001,0.001)$

Contaminant probability: $\operatorname{logit} c_{0} \sim \mathcal{N}(0,0.001), \tau_{c} \sim \Gamma(1.001,0.001)$

Residual time: $R_{0} \sim \mathcal{N}(0,0.001) \mathbb{T}(0,5), \tau_{R} \sim \Gamma(1.001,0.001)$

Decision parameters for each subject $s$ :

$$
M_{s} \sim \text { Categorical }(\pi), \log B_{s} \sim \mathcal{N}\left(\log B_{0}, \tau_{B}\right), \log A_{s} \sim \mathcal{N}\left(\log A_{0}, \tau_{A}\right)
$$

$\operatorname{logit} w_{s} \sim \mathcal{N}\left(\operatorname{logit} w_{0}, \tau_{w}\right), \operatorname{logit} c_{s} \sim \mathcal{N}\left(\operatorname{logit} c_{0}, \tau_{c}\right), R_{s} \sim \mathcal{N}\left(R_{0}, \tau_{R}\right) \mathbb{T}(0,5)$

Positive response boundary: $B_{s}^{+}=\left(1-w_{s}\right) B_{S}+A_{s}$

Negative response boundary: $B_{s}^{-}=w_{s} B_{S}+A_{s}$

Drift rates for item strengths $i$, associative strengths $j$, accumulators $k \in\{+,-\}$, subjects

$s$ :

$$
\begin{aligned}
& \forall . \tau . \sim \Gamma(1.001,0.001) \\
& d_{0} \sim \mathcal{N}(0,0.001) \\
& \forall_{i} d_{i} \sim \mathcal{N}\left(0, \tau_{I}\right), \forall_{j} d_{j} \sim \mathcal{N}\left(0, \tau_{J}\right), \forall_{k} d_{k} \sim \mathcal{N}\left(0, \tau_{K}\right), \forall_{s} d_{s} \sim \mathcal{N}\left(0, \tau_{S}\right) \\
& \forall_{i, j} d_{i j} \sim \mathcal{N}\left(0, \tau_{I J}\right), \forall_{i, k} d_{i k} \sim \mathcal{N}\left(0, \tau_{I K}\right), \forall_{i, s} d_{i s} \sim \mathcal{N}\left(0, \tau_{I S}\right), \forall_{j, k} d_{j k} \sim \mathcal{N}\left(0, \tau_{J K}\right) \\
& \forall_{j, s} d_{j s} \sim \mathcal{N}\left(0, \tau_{J S}\right), \forall_{k, s} d_{k s} \sim \mathcal{N}\left(0, \tau_{K S}\right) \\
& \forall_{i, j, k} d_{i j k} \sim \mathcal{N}\left(0, \tau_{I J K}\right), \forall_{i, j, s} d_{i j s} \sim \mathcal{N}\left(0, \tau_{I J S}\right), \forall_{i, k, s} d_{i k s} \sim \mathcal{N}\left(0, \tau_{I K S}\right) \\
& \forall_{j, k, s} d_{j k s} \sim \mathcal{N}\left(0, \tau_{J K S}\right) \\
& \forall_{i, j, k, s} d_{i j k s} \sim \mathcal{N}\left(0, \tau_{I J K S}\right) \\
& \text { Drift means: } d_{i j s}^{+}=d_{0}+d_{i}+d_{j}+d_{+}+d_{s}+d_{i j}+d_{i+}+d_{i s}+d_{j+}+d_{j s}+d_{+s} \\
& \quad+d_{i j+}+d_{i j s}+d_{i+s}+d_{j+s}+d_{i j+s} \\
& d_{i j s}^{-}= d_{0}+d_{i}+d_{j}+d_{-}+d_{s}+d_{i j}+d_{i-}+d_{i s}+d_{j-}+d_{j s}+d_{-s} \\
&+d_{i j-}+d_{i j s}+d_{i-s}+d_{j-s}+d_{i j-s}
\end{aligned}
$$

Joint likelihood for response $\mathrm{R}_{n} \in\{0,1\}$ at time $\mathrm{RT}_{n}\left(0.2<\mathrm{RT}_{n}<5\right)$, given item strength $I[n]$, associative strength $A[n]$, and subject $S[n]$ on trial $n$ :

$$
\begin{aligned}
\left\{\mathrm{R}_{n}, \mathrm{RT}_{n}\right\} \sim c_{S[n]} & \times \frac{0.75-0.5 \mathrm{R}_{n}}{5-0.2} \\
& +\left(1-c_{S[n]}\right) \times L_{M_{S[n]}}\left(\mathrm{R}_{n}, \mathrm{RT}_{n} \mid B_{S[n]}^{+}, B_{S[n]}^{-}, A_{S[n]}, R_{S[n]}, d_{I[n], A[n], S[n]}^{+}, d_{I[n], A[n], S[n]}^{-}\right)
\end{aligned}
$$

Figure D.1: Hierarchical model structure used to estimate LBA parameters and retrieval architectures. Note that we parameterize the response thresholds in terms of a "boundary separation" $B_{s}$ and "response bias" $w_{s}$. Likelihoods are a mixture of chance responding (first summand) and responding based on retrieval (second summand), as described in the main text. 

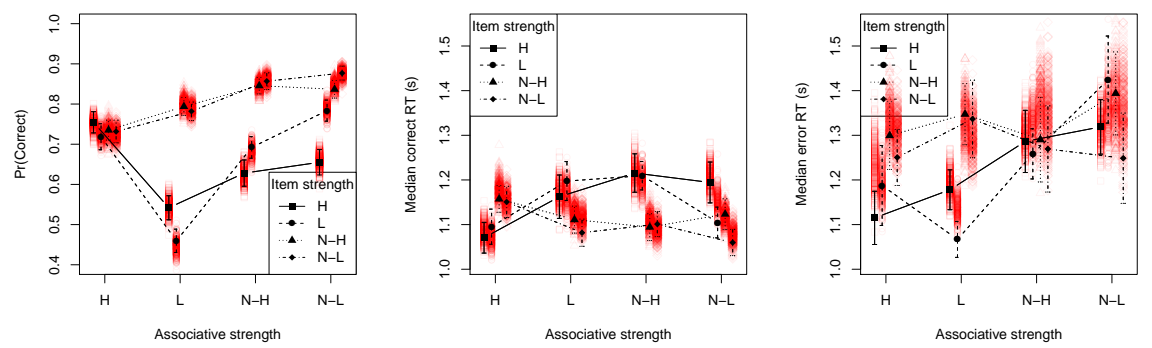

Figure D.2: Posterior predictive distributions from our hierarchical LBA model. Observed data are in black, showing means plus/minus 1.96 standard errors of the mean. Red points are posterior samples.

those things trade off with one another? In other words, is it possible to meaningfully distinguish between these two forms of interaction within this LBA modeling framework? To address this question, we also computed the posterior distribution over correlations between individual parameters. For each posterior sample, we computed the complete set of Pearson correlations between each participant's LBA parameters $\left(B_{s}, A_{s}, w_{s}, R_{s}, c_{s}\right.$, as well as the sum and difference of positive and negative drift rates for each pair type) and between a 1/0 indicator variable indicating which of the 7 possible retrieval processes was sampled for that participant. These correlations are depicted in Figure D.3

It is clear that retrieval speed - the sums of positive and negative drift rates for each pair type-are positively correlated within participants, as is retrieval accuracy (the difference between positive and negative drift rates in each condition). Pertinent to the current question, however, is that retrieval processing is not credibly correlated with retrieval accuracy and to the extent that it is correlated with retrieval speed, it does so across all pair types, rather than being specific to any one pair type (negative correlations between FEX and summed drift rates). In other words, to the extent that overall retrieval speed is reduced under facilitatory exhaustive processing, it does so evenly across all levels of item and associative strength and without also being correlated with retrieval accuracy. The identification of the majority of participants as engaging in facilitatory exhaustive processing is not an artifact of differing interactions between item and associative strength at encoding. 


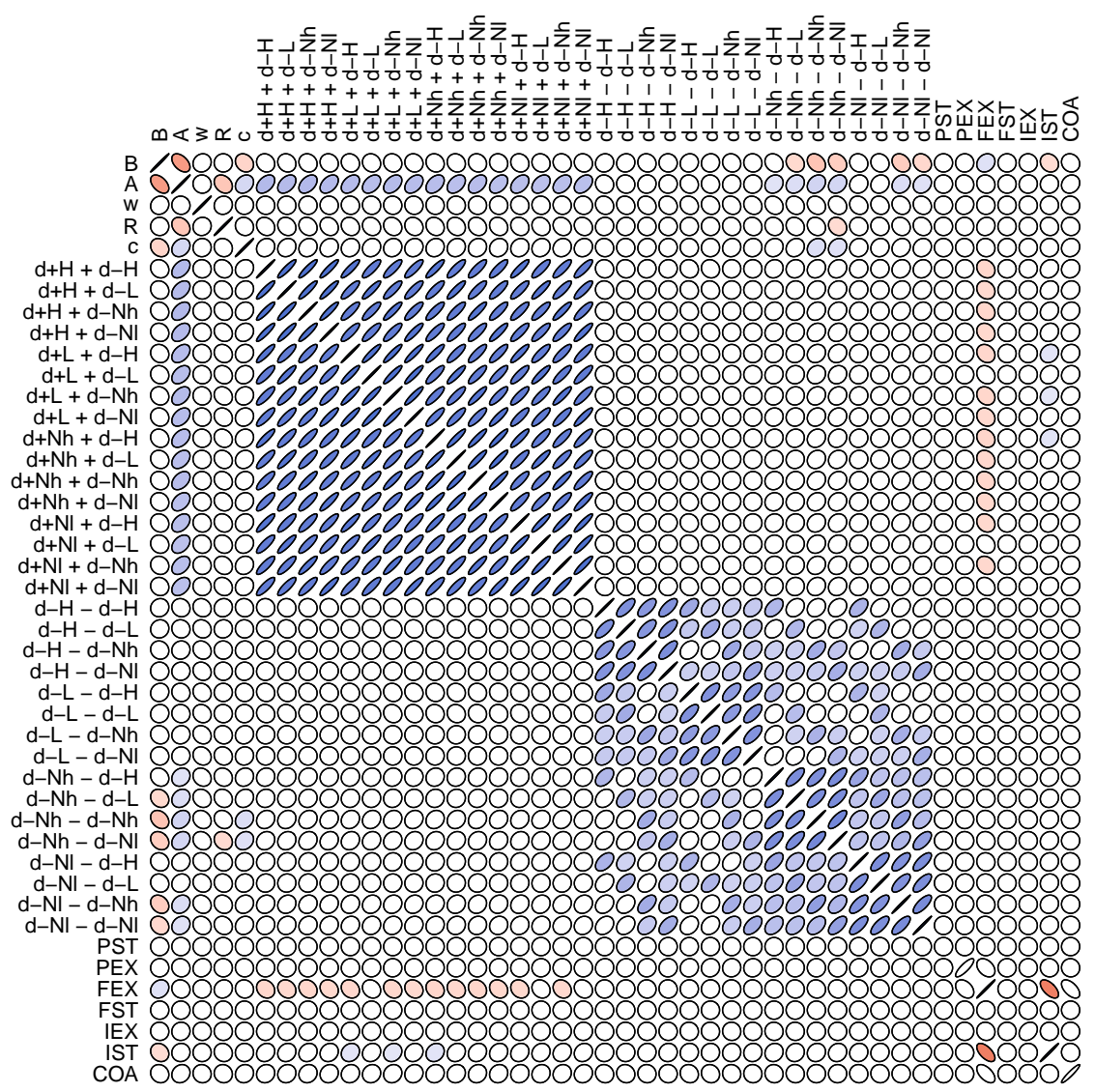

Figure D.3: Posterior distribution over individual-level correlations between LBA parameters. A non-filled ellipse indicates that the $95 \%$ credible interval for that correlation includes zero. Red indicates a credible negative correlation and blue indicates a credible positive correlation, with the width of the ellipse and the saturation of the color reflecting the strength of the correlation. Sums of drift means reflect retrieval speed while differences in drift rates reflect retrieval accuracy. Retrieval processing is labeled with respect to the processing leading toward positive responses: independent parallel self-terminating (PST), independent parallel exhaustive (PEX), facilitatory exhaustive (FEX), facilitatory self-terminating (FST), inhibitory exhaustive (IEX), inhibitory self-terminating (IST), and coactive (COA). 